\title{
A phase-field formulation for fracture in ductile materials: Finite deformation balance law derivation, plastic degradation, and stress triaxiality effects
}

\author{
Michael J. Borden ${ }^{1, *} \quad$ Thomas J. R. Hughes ${ }^{2,3} \quad$ Chad M. Landis ${ }^{2,3}$ \\ Amin Anvari $^{3} \quad$ Isaac J. Lee ${ }^{2}$ \\ ${ }^{1}$ Civil, Construction, and Environmental Engineering \\ North Carolina State University \\ Box 7908, Raleigh, North Carolina 27695 , USA \\ ${ }^{2}$ Institute for Computational Engineering and Science \\ The University of Texas at Austin \\ 201 East 24th St. Stop C0200, Austin, Texas 78712, USA \\ ${ }^{3}$ Aerospace Engineering and Engineering Mechanics \\ The University of Texas at Austin \\ 210 East 24th St. Stop C0600, Austin, Texas 78712, USA
}

\begin{abstract}
Phase-field models have been a topic of much research in recent years. Results have shown that these models are able to produce complex crack patterns in both two and three dimensions. A number of extensions from brittle to ductile materials have been proposed and results are promising. To date, however, these extensions have not accurately represented strains after crack initiation or included important aspects of ductile fracture such as stress triaxiality. This work introduces a number of contributions to further develop phase-field models for fracture in ductile materials. These contributions include: a cubic degradation function that provides a stress strain response prior to crack initiation that more closely approximates linear elastic behavior, a derivation of the governing equations in terms of a general energy potential from
\end{abstract}

*Corresponding author: mjborden@ncsu.edu

Preprint submitted to Computer Methods in Applied Mechanics and Engineering

(C) 2016. This manuscript version is made available under the Elsevier user license http://www.elsevier.com/open-access/userlicense/1.0/ 
balance laws that describe the kinematics of both the body and phase-field, introduction of a yield surface degradation function that provides a mechanism for plastic softening and corrects the non-physical elastic deformations after crack initiation, a proposed mechanism for including a measure of stress triaxiality as a driving force for crack initiation and propagation, and a correction to an error in the configuration update of an elastoplastic return-mapping algorithm for $J_{2}$ flow theory. We also present a heuristic time stepping scheme that facilitates computations that require a relatively long load time prior to crack initiation. A number of numerical results will be presented that demonstrate the effects of these contributions.

Keywords: Phase-field, fracture, plasticity, triaxiality

\section{Introduction}

The development of computational methods that accurately predict complex failure and fracture processes in ductile materials has proven difficult, particularly for full three-dimensional geometries, and many approaches have been proposed with varying success. The phase-field method, also known as the variational approach to fracture, is an approach that has recently received much attention in the literature. It has been shown that this method is able to produce complex crack patterns, including branching and merging, in both two and three dimensions. This approach leads to a system of partial differential equations that fully describe the evolution of crack surfaces without the need for ad hoc additions to the theory. Computations with this method are performed on the original mesh and no crack interface tracking is required. This leads to robust computational implementations. To date, most of the research has focused on brittle material. In this work we introduce additions and modifications to the theory that improve its ability to predict fracture in ductile materials.

The variational approach to brittle fracture, proposed by Francfort and Marigo [16], seeks to find a solution to the fracture problem by minimizing a potential energy based on Griffith's theory of brittle fracture. This approach leads to a Mumford-Shah [36] type energy potential that can be approximated by a phase-field formulation following the work of Ambrosio and Tortorelli [3]. This approximation was adopted by Bourdin et al. [10] to facilitate numerical solutions of the variational formulation. An alternative formulation, based on continuum mechanics and thermodynamic arguments, was presented by Miehe et al. [30] and Miehe et al. [29]. In addition to an alternative derivation, Miehe et al. [29] also added an important mechanism for distinguishing tensile and compressive effects on crack growth. The works of Larsen [25], Larsen et al. [26], Bourdin et al. [12], Borden et al. [8], and Hofacker and Miehe [21] have shown that this approach can be ex- 
tended to dynamic fracture and produces results that agree well with several benchmark problems. Other problems of interest that the variational approach to fracture has been applied to include hydraulic fracturing (Bourdin et al. [13], Mikelic̀ et al. [35]), piezoelectric ceramics (Wilson et al. [47]), rubbery polymers (Miehe and Schänzel [32]), and thermo-elastic solids (Miehe et al. [33]).

Initial work to extend the variational approach to ductile materials has been reported in Ambati et al. [1, 2] and Miehe et al. [31, 34]. The approach presented by Ambati et al. [1] augments the degradation function by a term that increases damage based on the accumulation of an equivalent plastic strain measure. Miehe et al. [31] have modified the history functional, $\mathcal{H}$, which drives the evolution of the phase-field, to include an equivalent plastic strain measure. A similar approach is presented by McAuliffe and Waisman [27] where a model that couples the phase-field with ductile shear band development is described. In this approach shear bands are modeled using an elasticviscoplastic model and fracture is modeled as the degradation of the volumetric elastic stress terms only (the deviatoric stress is undamaged).

In these previous approaches, however, the accumulation of plastic deformation reduces the elastic strength of the material in some way, but the plastic response is unmodified. This means that the yield surface and hardening modulus are not effected by the evolution of the phase-field. The result of this is that at some point the plastic strains saturate and the deformation becomes dominated by recoverable elastic strains. However, the experimental results presented in [17] show that ductile failure of an aluminum alloy is preceded by large plastic strains and that void nucleation and growth occurs in a relatively short strain interval just before failure. This behavior is contrary to what is predicted by the existing phase-field models for ductile materials.

In this work we present a number of contributions for phase-field models of fracture in ductile materials. These contributions include: 1) The introduction of a cubic degradation function that provides a stress-strain response prior to crack initiation that more closely approximates linear elastic behavior. 2) A microforce derivation of the governing equations in terms of a general energy potential for finite deformation problems. 3) The introduction of a yield surface degradation function that provides a mechanism for plastic softening and corrects the non-physical elastic deformations after crack initiation. 4) A mechanism for including a measure of stress triaxiality as a driving force for crack initiation and propagation. 5) A modification of the elastoplastic return mapping algorithm for $J_{2}$ flow theory presented by Simo and Hughes [43] that corrects an error in the update of the elastic configuration, which leads to errors in the calculation of the strain energy.

The paper will proceed as follows: In Section 2 we will establish the notation, describe the phase-field approach to fracture, introduce the cubic degradation function, and present a balance law derivation of the governing equations in terms of a general energy potential. In Section 3 
we will present the hyperelastic-plastic constitutive model that governs the stress response of a body and determines the contributing factors to crack growth. In this section we introduce new developments that couple crack growth with stress triaxiality and plastic softening. A summary of the constitutive model is given in Section 3.4. Section 4 presents the finite element formulation and establishes the return mapping algorithm. Numerical results are presented in Section 5. In this section we also present a heuristic adaptive time stepping scheme for cases with long loading times prior to crack initiation and propagation. In Section 6 we summarize our results and conclusions. A detailed description of the elastoplastic constitutive model and return-mapping algorithm are given in the appendix.

\section{Formulation}

In this section we develop a large deformation phase-field formulation for fracture in ductile materials. In this formulation, we will assume an energetic description of the fracture process and derive the governing equations from an energy balance principle.

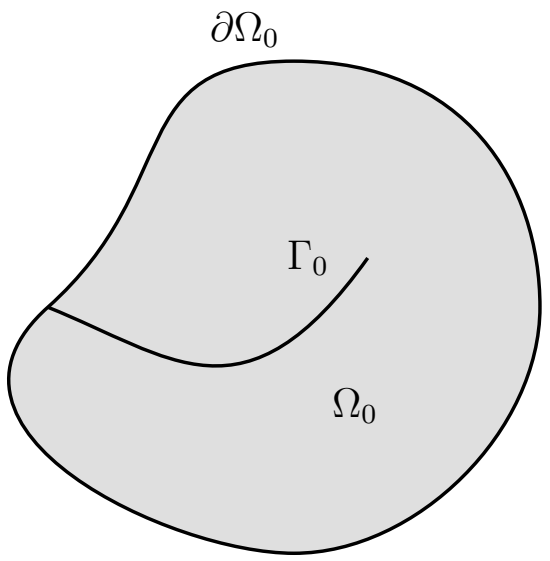

(a)

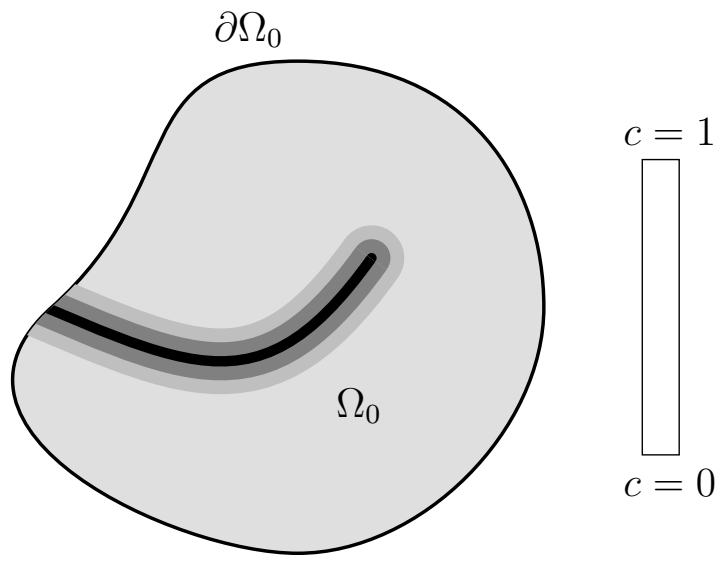

(b)

Figure 1: (a) A sketch of a body, $\Omega_{0}$, with an internal discontinuity boundary $\Gamma_{0}$. (b) The phasefield representation of the internal discontinuity.

\subsection{Kinematics}

We begin by establishing the notation that we will use to describe the kinematics of the body. We let $\Omega_{0} \subset \mathbb{R}^{d}$ (with $d \in\{2,3\}$ ) be the reference configuration of an arbitrary body with external boundary $\partial \Omega_{0}$ and evolving internal discontinuity boundary $\Gamma_{0}(t)$ as shown in Figure 1(a). We 
assume that the internal discontinuity evolves in an irreversible manner such that $\Gamma_{0}(t) \subset \Gamma_{0}(t+$ $\Delta t$ ) for all $\Delta t>0$. The motion of the body is described by the time dependent deformation map $\varphi:\left(\Omega_{0} \times \mathbb{R}\right) \rightarrow \mathbb{R}^{d}$, which maps a point $\mathbf{X} \in \Omega_{0}$ at time $t \in[0, T]$ to a point $\mathbf{x}=\varphi(\mathbf{X}, t) \in$ $\mathbb{R}^{d}$. The reference configuration is mapped to the current configuration through the motion as $\Omega_{t}=\varphi\left(\Omega_{0}, t\right)$ and the boundary of the current configuration is represented as $\partial \Omega_{t}$. The internal discontinuity is also mapped to the current configuration through the motion as $\Gamma_{t}=\varphi\left(\Gamma_{0}(t), t\right)$.

The deformation gradient is a second-order tensor defined as

$$
\mathbf{F}=\varphi, \mathbf{X}(\mathbf{X}, t)=\frac{\partial \varphi(\mathbf{X}, t)}{\partial \mathbf{X}}
$$

and the displacement of a point $\mathbf{X}$ at time $t$ is denoted by $\mathbf{u}(\mathbf{X}, t)=\varphi(\mathbf{X}, t)-\mathbf{X}$. The displacement field satisfies time-dependent Dirichlet boundary conditions, $u_{i}(\mathbf{X}, t)=g_{i}(\mathbf{X}, t)$, on $\partial \Omega_{g_{i}} \subseteq \partial \Omega_{t}$, and time-dependent Neumann boundary conditions on $\partial \Omega_{h_{i}} \subseteq \partial \Omega_{t}$.

For a ductile response, we assume the existences of a stress-free deformation from which the elastic deformation can be characterized. This assumption leads to a local multiplicative decomposition of the deformation gradient into elastic and plastic parts that takes the form

$$
\mathbf{F}=\mathbf{F}^{e} \mathbf{F}^{p}
$$

where $\mathbf{F}^{e}$ and $\mathbf{F}^{p}$ are the elastic and plastic deformations gradients respectively (see Simo and Hughes [43] for details). We denote derivatives with respect to the coordinates in the reference configuration as

$$
(\cdot), \mathbf{x}=\frac{\partial}{\partial \mathbf{X}}(\cdot) \quad \operatorname{DIV}(\cdot)=\frac{\partial}{\partial \mathbf{X}} \cdot(\cdot)
$$

and derivative with respect to the coordinates in the current configuration as

$$
(\cdot)_{\mathbf{x}}=\frac{\partial}{\partial \mathbf{x}}(\cdot), \quad \operatorname{div}(\cdot)=\frac{\partial}{\partial \mathbf{x}} \cdot(\cdot)
$$

\subsection{Phase-field approximations of fracture}

For energetic descriptions of fractured bodies, e.g. Griffith's theory of brittle fracture, the stored energy of a body can be written in the reference configuration as

$$
\Psi\left(\mathbf{F}, \Gamma_{0}\right)=\int_{\Omega_{0}} W(\mathbf{F}) d \Omega_{0}+\int_{\Gamma_{0}} \mathcal{G}_{c}^{0} d \Gamma_{0}
$$


where $W$ is the elastic strain energy per unit volume and $\mathcal{G}_{c}^{0}$ is the critical fracture energy per unit area in the reference configuration. For the case of brittle fracture, Francfort and Marigo [16] have proposed a variational approach that predicts crack evolution by finding a global minimizer of (5). To facilitate the numerical solution of this problem, Bourdin et al. [11] introduced a phase-field approximation of (5) that takes the form

$$
\tilde{\Psi}(\mathbf{F}, c, c, \mathbf{X})=\int_{\Omega_{0}} g(c) W(\mathbf{F}) d \Omega_{0}+\int_{\Omega_{0}} \mathcal{G}_{c}^{0} \Gamma_{c}(c, c, \mathbf{X}) d \Omega_{0}
$$

where $c \in[0,1]$ is the phase-field parameter, $g(c)$ is the degradation function that acts to reduce the elastic strength of the material, and

$$
\int_{\Omega_{0}} \mathcal{G}_{c}^{0} \Gamma_{c} d \Omega_{0} \approx \int_{\Gamma_{0}} \mathcal{G}_{c}^{0} d \Gamma_{0}
$$

is a volumetric approximation of the energy contribution from the crack surface. We call $\Gamma_{c}$ the crack density functional, which typically takes the form

$$
\Gamma_{c}(c, c, \mathbf{X})=\frac{1}{4 \ell_{0}}\left[(c-1)^{2}+4 \ell_{0}^{2}|c, \mathbf{X}|^{2}\right]
$$

where $\ell_{0} \in \mathbb{R}^{+}$is a length scale parameter that determines the width of the approximation. Other crack density functionals have been proposed, for example those by Burke et al. [15] and also a higher-order functional by Borden et al. [9].

Amor et al. [4], Miehe, Hofacker, and Welschinger [29], and Miehe, Welschinger, and Hofacker [30] further refined this approximation by introducing an additive decomposition of the elastic strain energy to distinguish between tensile and compressive contributions. The resulting approximation takes the form

$$
\tilde{\Psi}(\mathbf{F}, c, c, \mathbf{X})=\int_{\Omega_{0}}\left\{g(c) W^{+}(\mathbf{F})+W^{-}(\mathbf{F})\right\} d \Omega_{0}+\int_{\Omega_{0}} \mathcal{G}_{c}^{0} \Gamma_{c}(c, c, \mathbf{X}) d \Omega_{0}
$$

where $W^{+}$represents tensile contributions and $W^{-}$represents compressive contributions to the stored elastic strain energy. This modification provides a mechanism for distinguishing states of strain under which cracks will grow. For example, one popular decomposition introduced in [29] and [30] is based on a spectral decomposition of the strain tensor. In this form the sign of the principal strains are used to determine tensile and compressive contributions. Another approach proposed in [4] uses a constitutive model that decouples the volumetric and deviatoric response and defines the tensile contributions as the deviatoric part of the model. We will discuss these 
approaches in more detail in Section 3.1.

For the energy functional presented in (9) crack growth is driven by elastic strain energy. To extend this theory to ductile materials and provide a mechanism for plastic yielding to contribute to crack growth we add an effective plastic work contribution, $W_{p}$, to the stored energy. The stored energy functional that we propose is

$$
\begin{aligned}
\tilde{\Psi}\left(\mathbf{F}, c, c, \mathbf{X}, \mathbf{F}^{p}, \alpha\right) & =\int_{\Omega_{0}}\left\{g(c) W^{+}\left(\mathbf{F}, \mathbf{F}^{p}\right)+W^{-}\left(\mathbf{F}, \mathbf{F}^{p}\right)+g_{p}(c) W_{p}(\alpha)\right\} d \Omega_{0} \\
& +\int_{\Omega_{0}} \frac{\mathcal{G}_{c}^{0}}{4 \ell_{0}}\left[(c-1)^{2}+4 \ell_{0}^{2}|c, \mathbf{X}|^{2}\right] d \Omega_{0}
\end{aligned}
$$

where $\alpha$ is an internal hardening variable and $g_{p}(c)$ is the plastic degradation function. The plastic degradation function is analogous to the elastic degradation function and provides a mechanism for driving crack growth by the development of plastic strains.

\subsubsection{The cubic degradation function}

The stress degradation function, $g:[0,1] \rightarrow[0,1]$ plays an important role in the formulation because it determines how the stress responds to changes in the phase-field. A simple quadratic degradation function that is common in the literature is

$$
g(c)=c^{2}
$$

A plot of this function and corresponding stress-strain response for uniaxial homogeneous stress are shown as the dashed lines in Figure 2. We call the maximum stress the critical stress and the corresponding strain is called the critical strain. For the quadratic degradation function these values are

$$
\sigma_{c}^{\text {quad }}=\frac{9}{16} \sqrt{\frac{E \mathcal{G}_{c}^{0}}{6 \ell_{0}}}, \quad \varepsilon_{c}^{\text {quad }}=\sqrt{\frac{\mathcal{G}_{c}^{0}}{6 \ell_{0} E}} .
$$

It is clear that the critical stress will increase as $\ell_{0}$ decreases. In the limit as $\ell_{0}$ goes to zero, i.e., when the phase-field formulation coincides with the discrete fracture formulation, the critical stress goes to infinity.

The critical value for the phase-field, that is the value of the phase-field parameter when the stress is equal to the critical stress, is independent of the model and material parameters and is given by

$$
c_{c}=\sqrt{\frac{\sigma_{c}}{E \varepsilon_{c}}}=\frac{3}{4} .
$$



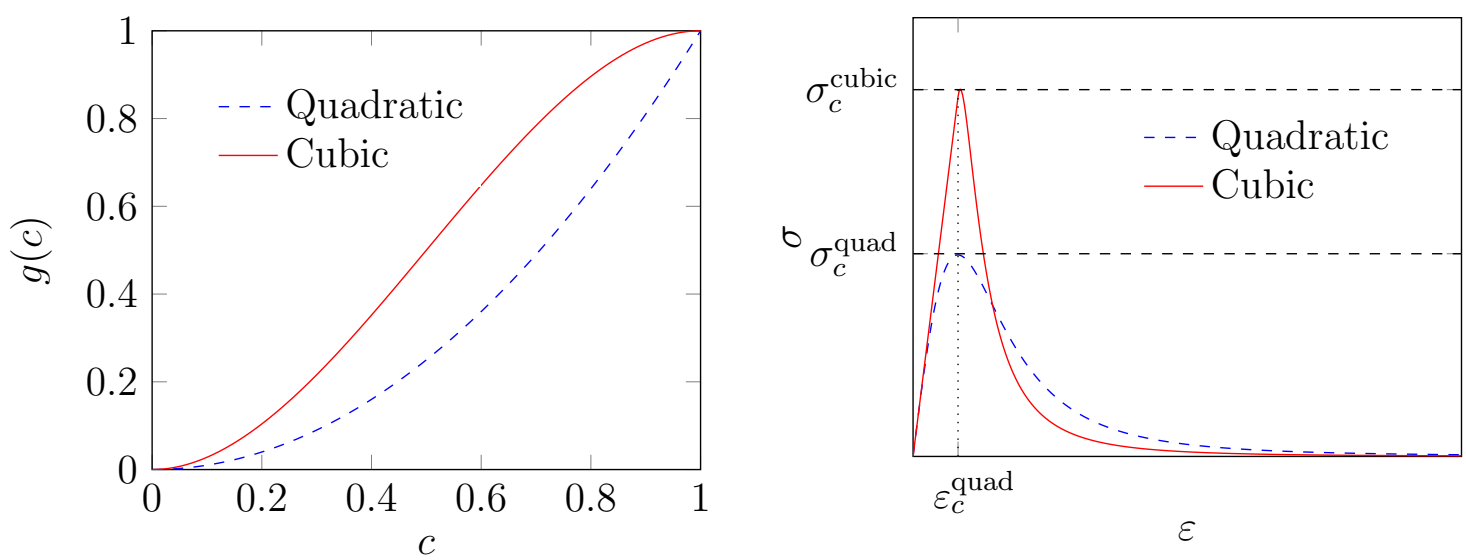

Figure 2: The quadratic and cubic degradation functions (left) and the respective stress-strain plots for a uniaxial homogeneous stress response (right). The material parameters are the same for both stress-strain responses.

This implies that, no matter what parameters are used, a 25 percent reduction in the phase-field is established prior to reaching the critical stress. It is also clear from the plot of the stress-strain curve that the response is nonlinear up to this point. This non-linear elastic stress-strain response effects material behavior prior to yielding in ductile materials as will be shown in Section 5.1. To address these issues we propose a parameterized cubic degradation function given by

$$
g(c)=m\left(c^{3}-c^{2}\right)+3 c^{2}-2 c^{3}
$$

where $m>0$ determines the slope of $g$ at $c=1$ (the quadratic degradation function is a special case of this function for $m=2$ ). Using this cubic degradation function, it can be shown that for $\varepsilon \leq \varepsilon_{c}^{\text {quad }}$

$$
\lim _{m \rightarrow 0} \sigma(\varepsilon)=E \varepsilon
$$

In other words, as $m \rightarrow 0$ the stress-strain relationship becomes linear up to the value of strain equal to the critical value found for the quadratic degradation function. This function and the resulting stress-strain curve for $m=10^{-4}$ are shown as the solid line in Figure 2. For comparison the critical stress and strain for the cubic degradation function are given in the limit as $m \rightarrow 0$ as

$$
\lim _{m \rightarrow 0} \sigma_{c}^{\text {cubic }}=\frac{81}{50} \sqrt{\frac{E \mathcal{G}_{c}^{0}}{15 \ell_{0}}}, \quad \lim _{m \rightarrow 0} \varepsilon_{c}^{\text {cubic }}=\sqrt{\frac{5 \mathcal{G}_{c}^{0}}{27 \ell_{0} E}}
$$


where the critical stress in this case is about 1.8 times higher than what is obtained with the quadratic degradation function and the critical strain is slightly higher (there is a small non-linear region for $\varepsilon_{c}^{\text {quad }}<\varepsilon<\varepsilon_{c}^{\text {cubic }}$.

The parameter $m$ in the cubic degradation function is used to set a positive but small slope at $c=1$. If the derivative of the degradation function is allowed to be zero at $c=1$ then a uniform phase-field $(\delta c=0)$ would satisfy the governing equations for any state of strain (see (47)) and crack nucleation would not occur. For all simulations presented in the following sections we have set $m=10^{-4}$, but do not consider it to be a model parameter with any physical meaning.

There are two points about the cubic degradation function we will emphasize. The first is the nearly linear stress-strain behavior prior to reaching the critical stress. This means that the behavior of the model more closely reproduces the response that is predicted by linear elastic fracture mechanics. The second difference is that for a given length scale, the critical stress for the cubic degradation is larger. This implies that to achieve a given critical stress, a larger length scale parameter will be used for the cubic degradation function. This allows a coarser mesh to be used and can reduce the computational cost for a given simulation.

To further show that the cubic degradation more closely reproduces the linear elastic fracture mechanics response we can compare crack opening stresses for the double cantilever beam problems shown in Figure 3. This configuration allows us to compare the response computed using the phase-field formulation with the response computed using a standard linear elastic fracture mechanics approach. Furthermore, under displacement controlled loading the crack growth is stable allowing us to examine and compare the stress states at various crack lengths. For this example, we compare the crack opening stress for a crack tip that is approximately at the midpoint of the geometry. These stresses are plotted in Figure 4. This plot shows that there is a process zone near the crack for both the quadratic and cubic degradation functions where the stress deviates from the classical stress singularity predicted by linear elastic fracture mechanics. It is clear that this process zone is smaller for the cubic degradation function (recall that the $\ell_{0}$ is the same for both cases) and that the stresses more accurately reproduce the stresses computed from linear elastic fracture mechanics. This plot also suggests that we can tailor the stresses in the process zone by modifying the degradation function and this may be an interesting area for future investigation.

Remark 2.1. A study examining the use of gradient damage models to approximate brittle fracture by Pham et al. [38] has noted the fact that the quadratic degradation function leads to a model with no linear elastic regime in the stress-strain response. This study has looked at the effect of changing both the degradation function and the form of the surface energy term and has shown that certain combinations result in a linear response up to failure. In our formulation we have restricted our 

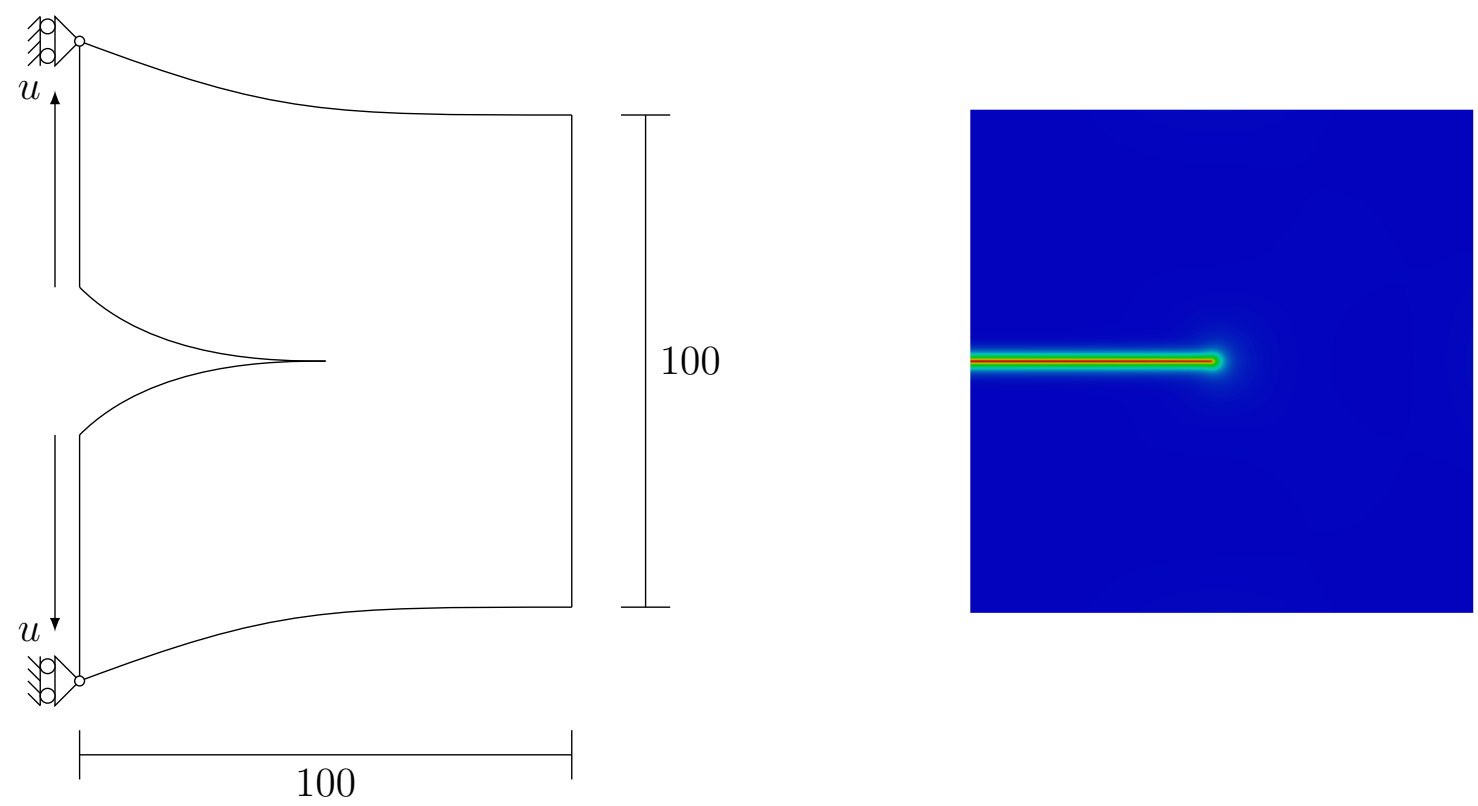

Figure 3: The geometry of the double cantilever beam is shown on the left and a phase-field representation of the crack is shown on the right.

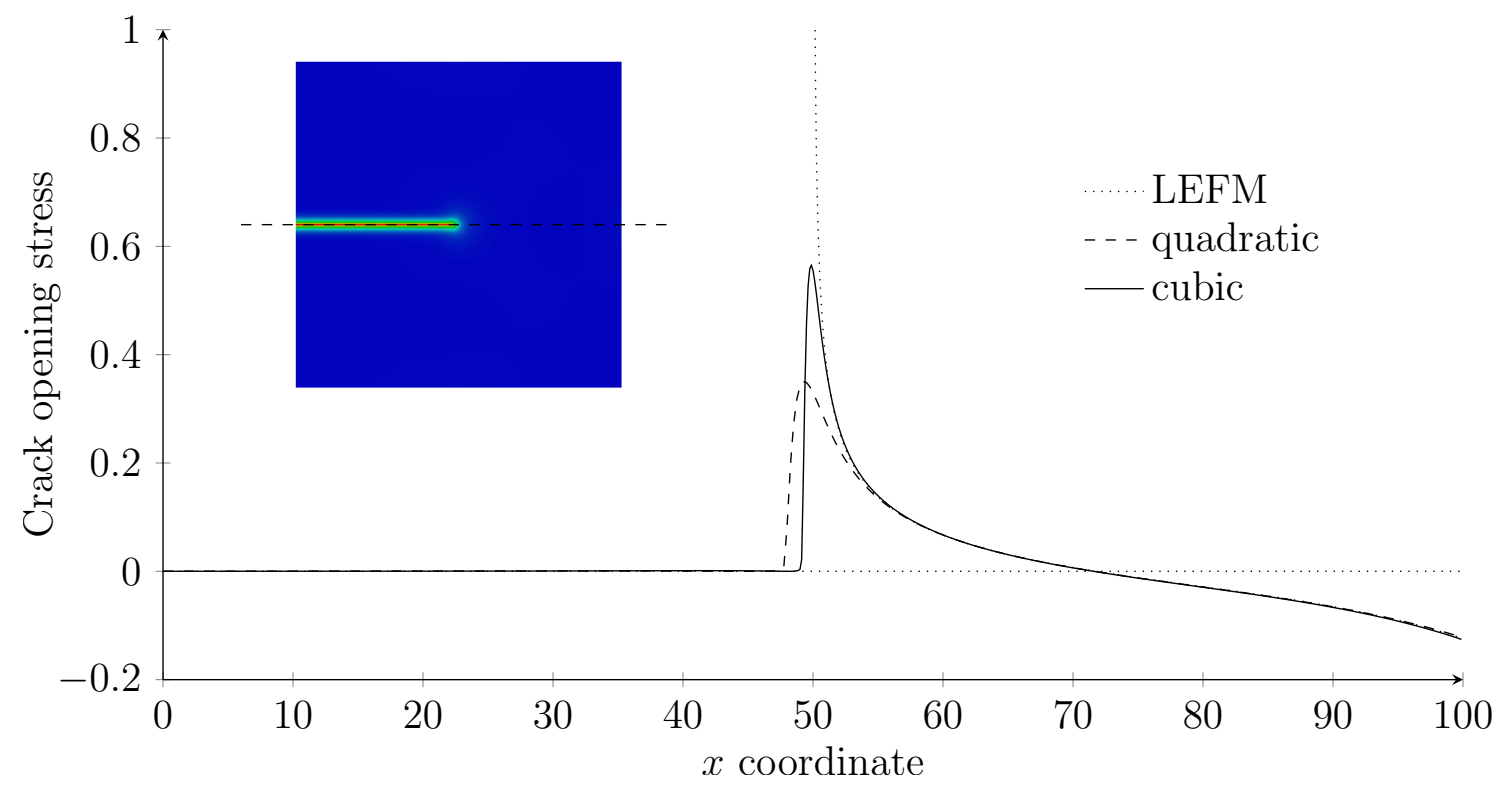

Figure 4: A plot of the crack opening stress for cracks computed using the phase-field approach with the quadratic and cubic degradation function and using a standard linear elastic fracture mechanics (LEFM) approach. $\ell_{0}$ is taken to be the same for the quadratic and cubic degradation function calculations. 
modifications to the degradation function and have left the surface energy unchanged.

\subsection{Balance law derivation of the phase-field fracture model}

In this section we postulate the existence of a microforce balance law that describes the kinematics of the phase-field. This balance law supplements the usual linear and angular momentum balance laws and provides a theory that allows a general, thermodynamically consistent derivation of the governing equations for phase-field models of fracture. This derivation separates balance laws from constitutive behavior. It also provides a natural way to introduce dissipation mechanisms through a general analysis of the second law of thermodynamics.

Microforce theories have been applied to other phase-field models. For example, Gurtin [19] has derived the Cahn-Hilliard equation from a microforce balance law. In addition, Stumpf and Hackl [44] introduced a microforce derivation for damage laws that provides a thermodynamically consistent framework for thermo-viscoelastic and quasi-brittle materials. More recently, a phasefield model for fracture in piezoelectric ceramics has been derived by Wilson et al. [47] based on a microforce approach. We follow the developments of this later work to derive a formulation in the large deformation setting restricted to purely mechanical processes.

We begin the derivation of the phase-field model with the usual linear and angular momentum balance laws. In order to localize the balance laws, we assume that the following integral equations hold for all $\mathcal{U} \subset \Omega_{0}$, where $\mathcal{U}$ is an open set with Lipshitz boundary $\partial \mathcal{U}$, and we assume all integrands are at least $C^{0}$-continuous functions. The linear and angular momentum balance laws are

\section{Linear momentum:}

$$
\begin{aligned}
& \frac{\mathrm{d}}{\mathrm{d} t} \int_{\mathcal{U}} \rho_{0} \dot{\mathbf{U}} d \mathcal{U}=\int_{\mathcal{U}} \mathbf{B} d \mathcal{U}+\int_{\partial \mathcal{U}} \mathbf{P N} d \partial \mathcal{U} \\
& \Rightarrow \operatorname{Div} \mathbf{P}+\mathbf{B}=\rho_{0} \ddot{\mathbf{U}}
\end{aligned}
$$

\section{Angular momentum:}

$$
\begin{aligned}
& \frac{\mathrm{d}}{\mathrm{d} t} \int_{\mathcal{U}} \mathbf{x} \times \rho_{0} \dot{\mathbf{U}} d \mathcal{U}=\int_{\mathcal{U}} \mathbf{x} \times \mathbf{B} d \mathcal{U}+\int_{\partial \mathcal{U}} \mathbf{x} \times(\mathbf{P N}) d \partial \mathcal{U} \\
& \Rightarrow \mathbf{P F}^{T}=\mathbf{F P}^{T}
\end{aligned}
$$


where $\rho_{0}$ is the mass density in the reference configuration, $\mathbf{P}$ is the first Piola-Kirchhoff stress tensor, $\mathbf{N}$ is the outward unit normal of $\partial \mathcal{U}, \mathbf{B}$ is the body force, $\mathbf{U}$ is the displacement, and the superposed dot is the material time derivative.

To derive the microforce balance law, we assume that at each time step the phase-field can be characterized by an internal microforce $\pi(\mathbf{x}, t) \in \mathbb{R}$, an external microforce $l(\mathbf{x}, t) \in \mathbb{R}$ acting on the body, and an external microforce $\lambda(\mathbf{x}, t) \in \mathbb{R}$ acting on the surface of the body. We also assume the existence of a micro force traction vector, $\boldsymbol{\xi}(\mathbf{x}, t) \in \mathbb{R}^{d}$, such that $\lambda=\boldsymbol{\xi} \cdot \mathbf{N}$ on $\partial \mathcal{U}$. Furthermore, we assume that there are no inertial terms associated with the microforces. Given these assumptions, we postulate the microforce balance law to be

\section{Microforce:}

$$
\begin{aligned}
& \int_{\partial \mathcal{U}} \boldsymbol{\xi} \cdot \mathbf{N} d \partial \mathcal{U}+\int_{\mathcal{U}} l d \mathcal{U}+\int_{\mathcal{U}} \pi d \mathcal{U}=0 \\
& \Rightarrow \operatorname{Div} \boldsymbol{\xi}+\pi+l=0
\end{aligned}
$$

Given these balance laws, the thermodynamic analysis of the phase-field fracture model follows:

Energy Balance Letting $e$ denote the internal energy per unit mass density and noting that $\dot{c}$ is rate-of-work conjugate to the microforces, the energy balance is stated as

$$
\begin{aligned}
& \frac{\mathrm{d}}{\mathrm{d} t} \int_{\mathcal{U}}\left(\frac{1}{2} \rho_{0}|\dot{\mathbf{U}}|^{2}+\rho_{0} e\right) d \mathcal{U} \\
& =\int_{\partial \mathcal{U}}(\mathbf{P N}) \cdot \dot{\mathbf{U}} d \partial \mathcal{U}+\int_{\mathcal{U}} \mathbf{B} \cdot \dot{\mathbf{U}} d \mathcal{U}+\int_{\partial \mathcal{U}}(\boldsymbol{\xi} \cdot \mathbf{N}) \dot{c} d \partial \mathcal{U}+\int_{\mathcal{U}} l \dot{c} d \mathcal{U} \\
& \Rightarrow \rho_{0} \dot{e}=\mathbf{P}: \dot{\mathbf{F}}+\boldsymbol{\xi} \cdot \dot{c}_{, \boldsymbol{X}}-\pi \dot{c} .
\end{aligned}
$$

Second law Let $s$ be the entropy per unit mass density. Then the second law of thermodynamics can be stated as

$$
\begin{aligned}
& \frac{\mathrm{d}}{\mathrm{d} t} \int_{\mathcal{U}} \rho_{0} s d \mathcal{U} \geq-\int_{\partial \mathcal{U}} \frac{\mathbf{q} \cdot \mathbf{N}}{\Theta} d \partial \mathcal{U}+\int_{\mathcal{U}} \frac{\rho_{0} r}{\Theta} d \mathcal{U} \\
& \Rightarrow \Theta \rho \dot{s} \geq \frac{1}{\Theta} \mathbf{q} \cdot \Theta_{, \boldsymbol{x}}-\operatorname{div} \mathbf{q}+\rho r
\end{aligned}
$$

where $\Theta$ is absolute temperature, $\mathbf{q}$ is the heat flux, and $r$ is a heat source. For the formulation presented here we assuming $r=0, \operatorname{Div} \mathbf{q}=0$, and $\Theta_{, \boldsymbol{X}}=\mathbf{0}$. Under these assumptions, the second 
law inequality simplifies to

$$
\Theta \rho_{0} \dot{s} \geq 0
$$

Dissipation inequality Using the Legendre transformation we write the internal energy in terms of the Helmholtz free energy and entropy as

$$
\rho_{0} e=\rho_{0} \psi+\Theta \rho_{0} s
$$

The dissipation inequality follows from this equation and is given by

$$
\rho_{0} \dot{\psi} \leq \rho_{0} \dot{e}
$$

Substituting (24) into (29), the dissipation inequality is written in terms of the Piola-Kirchhoff stress tensors and microforces as

$$
\rho_{0} \dot{\psi} \leq \mathbf{P}: \dot{\mathbf{F}}+\boldsymbol{\xi} \cdot \dot{c}_{, \mathbf{X}}-\pi \dot{c}
$$

or

$$
\rho_{0} \dot{\psi} \leq \frac{1}{2} \mathbf{S}: \dot{\mathbf{C}}+\boldsymbol{\xi} \cdot \dot{c}_{, \mathbf{X}}-\pi \dot{c}
$$

where $\mathbf{C}=\mathbf{F}^{T} \mathbf{F}$ is the right Cauchy-Green deformation tensor and $\mathbf{S}=\mathbf{F}^{-1} \mathbf{P}$ is the second Piola-Kirchhoff stress tensor.

This derivation provides a general formulation for phase-field fracture models. As long as the free energy, $\psi$, is defined such that (30) and (31) hold the model will be thermodynamically consistent.

\subsubsection{Governing equations}

To derive the governing equations we assume the Helmholtz free energy function takes the general form $\psi=\psi\left(\mathbf{C}, \mathbf{C}^{p}, \mathbf{Q}, c, c, \mathbf{X}, \dot{c}\right)$, where $\mathbf{Q}$ is a set of internal plastic variables and

$$
\mathbf{C}^{p}=\mathbf{F}^{p T} \mathbf{F}^{p}
$$


is the plastic right Cauchy-Green deformation tensor. Substituting this into (31) and integrating over the reference domain we get

$$
\begin{aligned}
& \int_{\Omega_{0}}\left(\rho_{0} \frac{\partial \psi}{\partial \mathbf{C}}: \dot{\mathbf{C}}+\rho_{0} \frac{\partial \psi}{\partial \mathbf{C}^{p}}: \dot{\mathbf{C}}^{p}+\rho_{0} \frac{\partial \psi}{\partial \mathbf{Q}} \cdot \dot{\mathbf{Q}}+\rho_{0} \frac{\partial \psi}{\partial c} \dot{c}+\rho_{0} \frac{\partial \psi}{\partial c, \mathbf{X}} \cdot \dot{c}_{, \mathbf{X}}+\rho_{0} \frac{\partial \psi}{\partial \dot{c}} \ddot{c}\right) d \Omega_{0} \\
& \leq \int_{\Omega_{0}}\left(\frac{1}{2} \mathbf{S}: \dot{\mathbf{C}}+\boldsymbol{\xi} \cdot \dot{c}_{, \mathbf{X}}-\pi \dot{c}\right) d \Omega_{0} .
\end{aligned}
$$

We now wish to relate terms on the left hand side of the inequality with terms on the right hand side. We begin by noting that for purely elastic deformations, we can assume that the plastic dissipation, defined as

$$
\mathbb{D}^{p}\left(\mathbf{C}, \mathbf{C}^{p}, \mathbf{Q} ; \dot{\mathbf{C}}^{p}, \dot{\mathbf{Q}}\right)=-\rho_{0} \frac{\partial \psi}{\partial \mathbf{C}^{p}}: \dot{\mathbf{C}}^{p}-\rho_{0} \frac{\partial \psi}{\partial \mathbf{Q}} \cdot \dot{\mathbf{Q}}
$$

is zero. The remaining terms on the left hand side are linear in $\dot{\mathbf{C}}, \dot{c}, \dot{c}_{, \mathbf{X}}$, and $\ddot{c}$ and we assume that the inequality must hold for arbitrary time histories of these variables. This leads to the following relations:

$$
\begin{aligned}
\rho_{0} \frac{\partial \psi}{\partial \dot{c}} & =0 \\
\rho_{0} \frac{\partial \psi}{\partial c_{,} \mathbf{X}} & =\boldsymbol{\xi} \\
2 \rho_{0} \frac{\partial \psi}{\partial \mathbf{C}} & =\mathbf{S} \\
\left(\pi+\rho_{0} \frac{\partial \psi}{\partial c}\right) \dot{c} \leq 0 & \Rightarrow \pi=-\rho_{0} \frac{\partial \psi}{\partial c}-\beta \dot{c} \\
\mathbb{D}^{p}=-\rho_{0} \frac{\partial \psi}{\partial \mathbf{C}^{p}}: \dot{\mathbf{C}}^{p}-\rho_{0} \frac{\partial \psi}{\partial \mathbf{Q}} \cdot \dot{\mathbf{Q}} & \geq 0
\end{aligned}
$$

where $\beta=\beta\left(\mathbf{C}, \mathbf{C}^{p}, c, c, \mathbf{X}\right) \geq 0$ a function that can be used to account for other dissipation mechanisms. Substituting these equations into the balance laws using $\mathbf{P}=\mathbf{F S}$ we get:

\section{Linear momentum:}

$$
\operatorname{Div}\left(2 \mathbf{F} \rho_{0} \frac{\partial \psi}{\partial \mathbf{C}}\right)+\mathbf{B}=\rho_{0} \ddot{\mathbf{U}}
$$

\section{Microforce:}

$$
\operatorname{Div}\left(\rho_{0} \frac{\partial \psi}{\partial c, \mathbf{X}}\right)+l-\rho_{0} \frac{\partial \psi}{\partial c}=\beta \dot{c}
$$


With the governing equations of motion and the second-order approximation from Section 2.2 in hand, we can derive the strong form of the phase-field model for ductile fracture. It follows from (10) that the Helmholtz free-energy is given by

$\rho_{0} \psi\left(\mathbf{C}, \mathbf{C}^{p}, \mathbf{Q}, c, c, \mathbf{X}\right)=g(c) W^{+}\left(\mathbf{C}, \mathbf{C}^{p}\right)+W^{-}\left(\mathbf{C}, \mathbf{C}^{p}\right)+g_{p}(c) W_{p}(\mathbf{Q})+\mathcal{G}_{c}^{0}\left[\frac{(1-c)^{2}}{4 \ell_{0}}+\ell_{0} c_{, \mathbf{X}} \cdot c_{, \mathbf{X}}\right]$

where we have left the energy density functions $W^{+}, W^{-}$, and $W_{p}$ to be defined later. Then

$$
\begin{aligned}
\operatorname{Div}\left(2 \mathbf{F} \rho_{0} \frac{\partial \psi}{\partial \mathbf{C}}\right) & =\operatorname{Div}\left(2 \mathbf{F}\left(g(c) \frac{\partial W^{+}}{\partial \mathbf{C}}+\frac{\partial W^{-}}{\partial \mathbf{C}}\right)\right) \\
\operatorname{Div}\left(\rho_{0} \frac{\partial \psi}{\partial c, \mathbf{X}}\right) & =2 \mathcal{G}_{c}^{0} \ell_{0} \operatorname{Div}\left(c_{, \mathbf{X}}\right) \\
\rho_{0} \frac{\partial \psi}{\partial c} & =g^{\prime} W^{+}+g_{p}^{\prime} W_{p}-\mathcal{G}_{c}^{0} \frac{1-c}{2 \ell_{0}} .
\end{aligned}
$$

substituting these back into (40) and (41) and taking $l=0$ and $\beta=0$ we get the following governing equations

$$
\begin{aligned}
& \operatorname{Div}\left(2 \mathbf{F}\left(g(c) \frac{\partial W^{+}}{\partial \mathbf{C}}+\frac{\partial W^{-}}{\partial \mathbf{C}}\right)\right)+\mathbf{B}=\rho_{0} \ddot{\mathbf{U}} \\
& \frac{2 \ell_{0}}{\mathcal{G}_{c}^{0}}\left(g^{\prime} W^{+}+g_{p}^{\prime} W_{p}\right)+c-4 \ell_{0}^{2} c, \mathbf{X} \cdot c_{, \mathbf{X}}=1 .
\end{aligned}
$$

Irreversibility of crack growth due to elastic deformation will be enforced through the history functional introduced by Miehe et al. [29], i.e.

$$
\mathcal{H}\left(\mathbf{C}, \mathbf{C}^{p}\right)=\max _{\hat{t} \leq t} W^{+}\left(\mathbf{C}(\hat{t}), \mathbf{C}^{p}(\hat{t})\right)
$$

Since it is assumed that the effective plastic work, $W_{p}$, is monotonically increasing no constraints are needed to enforce irreversible crack growth due to plastic deformation. However, in order to have more control of the contribution of plastic deformation to crack growth we introduce a plastic work threshold, $W_{0}$, to the formulation and replace $W_{p}$ in (47) with $\left\langle W_{p}-W_{0}\right\rangle$ where the angle bracket operator is defined as

$$
\langle x\rangle= \begin{cases}0 & x<0 \\ x & x \geq 0\end{cases}
$$


This modification provides a mechanism to control the point at which plastic deformation begins to contribute to crack growth (more details will be provided in Section 5.1). We also introduce two parameters $\beta_{e} \in[0,1]$ and $\beta_{p} \in[0,1]$ that may be used to weight the contribution to crack growth from elastic strain energy and plastic work. Substituting these modifications into (47) the updated phase-field equation becomes

$$
\frac{2 \ell_{0}}{\mathcal{G}_{c}^{0}}\left(\beta_{e} g^{\prime} \mathcal{H}+\beta_{p} g_{p}^{\prime}\left\langle W_{p}-W_{0}\right\rangle\right)+c-4 \ell_{0}^{2} c, \mathbf{X} \cdot c_{, \mathbf{X}}=1 .
$$

The governing equations are summarized in Section 3.4.1

\section{Large deformation constitutive response}

In Section 2 we have derived the strong form equations in terms of the general energy functionals $W^{+}, W^{-}$, and $W_{p}$. In this section we describe the constitutive relations we use to complete the formulation. This will include a finite deformation hyperelastic constitutive model, an elastoplastic constitutive model based on $J_{2}$ flow theory with isotropic hardening, and the effective plastic work driving energy term that takes into account the state of stress triaxiality.

\subsection{Finite deformation hyperelastic model}

The constitutive model governing the elastic response will be based on the assumption that the strain energy can be decomposed into volumetric and deviatoric contributions. We follow the strain energy decomposition introduced by Amor et al. [4] and define the tensile and compressive contributions to be

$$
\begin{aligned}
& W^{+}= \begin{cases}U\left(J^{e}\right)+\bar{W}\left(\overline{\mathbf{C}}, \mathbf{C}^{p}\right) & J^{e} \geq 1 \\
\bar{W}\left(\overline{\mathbf{C}}, \mathbf{C}^{p}\right) & J^{e}<1\end{cases} \\
& W^{-}= \begin{cases}0 & J^{e} \geq 1 \\
U\left(J^{e}\right) & J^{e}<1\end{cases}
\end{aligned}
$$

respectively, where

$$
J^{e}=\operatorname{det} \mathbf{F}^{e}
$$


and

$$
\overline{\mathbf{C}}=J^{e-2 / 3} \mathbf{C} .
$$

The damaged elastic strain energy is then given by

$$
W\left(J^{e}, \overline{\mathbf{C}}, \mathbf{C}^{p}, c\right)=g(c) W^{+}\left(J^{e}, \overline{\mathbf{C}}, \mathbf{C}^{p}\right)+W^{-}\left(J^{e}\right) .
$$

For the formulation presented here, we use the specific forms

$$
\begin{aligned}
U\left(J^{e}\right) & =\frac{1}{2} \kappa\left[\frac{1}{2}\left(J^{e 2}-1\right)-\ln J^{e}\right], \\
\bar{W}\left(\overline{\mathbf{C}}, \mathbf{C}^{p}\right) & =\frac{1}{2} \mu\left(\overline{\mathbf{C}}: \mathbf{C}^{p-1}-3\right)
\end{aligned}
$$

where $\kappa$ and $\mu$ are the bulk and shear modulus respectively. The resulting constitutive model for the elastic response is summarized in Section 3.4.2.

An alternative constitutive model that has been used in large deformation phase-field formulations is based on the spectral decomposition of the logarithmic strain tensor. Letting $\varepsilon^{e}$ represent the elastic logarithmic strains and

$$
\varepsilon^{e}=\sum \varepsilon_{a}^{e} \mathbf{n}_{a} \otimes \mathbf{n}_{a}
$$

be its spectral decomposition, we define the tensile part as

$$
\varepsilon^{e+}=\sum\left\langle\varepsilon_{a}^{e}\right\rangle \mathbf{n}_{a} \otimes \mathbf{n}_{a}
$$

and the compressive part as $\varepsilon^{e-}=\varepsilon^{e}-\varepsilon^{e+}$. The strain energy contributions are then given as

$$
\begin{aligned}
& W^{+}=\frac{1}{2} \lambda\left\langle\operatorname{tr} \varepsilon^{e}\right\rangle^{2}+\mu \varepsilon^{e+}: \varepsilon^{e+} \\
& W^{-}=\frac{1}{2} \lambda\left\langle-\operatorname{tr} \varepsilon^{e}\right\rangle^{2}+\mu \varepsilon^{e-}: \varepsilon^{e-}
\end{aligned}
$$

Although this representation leads to a split that tends to produce better results in certain brittle fracture simulations it leads to a more complex return mapping algorithm when simulating fracture in ductile materials. In our experience this has resulted in a loss of numerical accuracy and convergence problems and we will not consider its use in this paper. However, we intend to explore this issue in future work. 


\subsection{Elastoplastic constitutive model}

In this section we describe an associative elastoplastic constitutive model based on $J_{2}$ flow theory with isotropic hardening. We assume an isotropic stress response governed by the damage elastic constitutive model given in Section 3.4.2. We also assume the plastic flow to be isochoric, i.e.,

$$
\operatorname{det} \mathbf{F}^{p}=1 \quad \Rightarrow \quad J=\operatorname{det} \mathbf{F}^{e} .
$$

The standard yield function for $J_{2}$ plasticity is given by

$$
f(\boldsymbol{\tau}, \alpha)=\|\mathbf{s}\|-\sqrt{\frac{2}{3}} k(\alpha), \quad \mathbf{s}=\operatorname{dev} \boldsymbol{\tau}
$$

where $\alpha$ is the hardening parameter and $k(\alpha)$ the isotropic hardening modulus. The hardening law is determined by the choice of $k(\alpha)$ as, for example,

$$
k(\alpha)= \begin{cases}\sigma_{y} & (\text { Perfect plasticity) } \\ \sigma_{y}+K \alpha & \text { (Linear hardening) }\end{cases}
$$

where $\boldsymbol{\tau}=J^{e} \boldsymbol{\sigma}$ is the Kirchhoff stress, $\sigma_{y}$ is the yield stress, and $K$ is the linear hardening coefficient.

In Section 5.1 we will show that the standard yield function shown in (63) does not produce the correct physical response when coupled with the phase-field model for crack growth. As the material fails the damaged elastic response pulls the stress back within the yield surface and any further deformation up to complete failure is purely elastic (i.e. recoverable). This is contrary to what is observed physically for ductile materials where the deformation is dominated by plastic strain. To compensate for this behavior we multiply the yield surface by the plastic degradation function, $g_{p}(c)$. The modified yield function that we propose is

$$
f(\boldsymbol{\tau}, \alpha)=\|\mathbf{s}\|-g_{p}(c) \sqrt{\frac{2}{3}} k(\alpha) .
$$

A detailed study of this modified yield function will be presented in Section 5.1.

The elastoplastic constitutive model is completed by the associative flow rule, which is derived assuming maximum plastic dissipation. Under this assumption, and given the constitutive model summarized in Section 3.4.2 and yield function (65), the flow rule is

$$
\operatorname{dev}\left[L_{v} \mathbf{b}^{e}\right]=-\frac{2}{3} \dot{\gamma} \operatorname{tr}\left[\mathbf{b}^{e}\right] \mathbf{n}
$$




$$
\mathbf{n}=\frac{\mathbf{s}}{\|\mathbf{s}\|}
$$

where $\mathbf{b}^{e}=\mathbf{F}^{e} \mathbf{F}^{e T}, L_{v} \mathbf{b}^{e}$ is the Lie derivative of $\mathbf{b}^{e}$ (see Simo and Hughes [43] for details), and $\dot{\gamma}$ is the plastic multiplier from the constrained minimization problem that follows from the principle of maximum plastic dissipation. We note that the principle of maximum plastic dissipation determines only the deviatoric part of $L_{v} \mathbf{b}^{e}$. The evolution of the spherical part of $\mathbf{b}^{e}$ must be determined by the isochoric assumption, i.e. $\operatorname{det}\left[\mathbf{b}^{e}\right]=J^{2}$. A detailed derivation of the elastic/plastic constitutive model is presented in Appendix A and a brief summary is given in Section 3.4.3.

\subsection{Effective plastic work driving energy and stress triaxiality}

We take two approaches for computing the effective plastic work contribution to (50). In the first approach the effective plastic work is defined by the rate equation

$$
\dot{W}_{p}=\dot{\gamma}\|\mathbf{s}\|
$$

This approach provides a simple measure of the effective plastic work but provides no information about the state of stress.

In the second approach we modify the rate equation (68) to include information about stress triaxiality. It has long been established that the failure of ductile materials can be characterized by a combination of the effective plastic strain and the level of stress triaxiality. Material ductility as quantified by the equivalent strain to failure is higher for stress states when the ratio of the mean stress to the effective stress is low than when this measure of triaxiality is high. The microscopic mechanism of void growth responsible for this behavior was modeled in the seminal works of Bridgman [14], Rice and Tracey [39], and McClintock [28]. Thereafter, the Gurson-TvergaardNeedleman (GTN) plasticity model (Gurson [18], Tvergaard and Needleman [45]), which includes the volume fraction of voids as an internal variable, was established and has been used extensively to model ductile failure of metals. It is of note that the failure behavior at low triaxiality levels has received renewed interest due to the fact that the GTN model does not perform well in this regime, (Bao and Wierzbicki [7], Bai and Wierzbicki [5, 6], Halton et al. [20]). In this work it is recognized that the effects of triaxiality play a key role in the failure of ductile metals and must be incorporated into any phenomenological model that attempts to capture the physics of ductile failure. The recent works referenced above indicate that a full phenomenological description should incorporate triaxialty as well as the third invariant of the deviatoric stress tensor, however for the sake of simplicity the present modeling approach will implement the simpler failure criterion due 
to Johnson and Cook [24]. We note that the present framework allows for a more detailed failure criterion once established.

In this work stress triaxiality is measured in terms of the Kirchhoff stress as

$$
\frac{\tau_{m}}{\|\mathbf{s}\|}
$$

where

$$
\tau_{m}=\frac{\operatorname{tr}[\boldsymbol{\tau}]}{3}
$$

This measure of stress triaxiality is then incorporated into the measure of effective plastic work by modifying the rate equation for $\dot{W}_{p}$ such that

$$
\begin{aligned}
\dot{W}_{p} & =\dot{\gamma} \frac{\|\mathbf{s}\|}{\varphi} \\
\varphi & =d_{1}+d_{2} \exp \left[d_{3} \frac{\tau_{m}}{\|\mathbf{s}\|}\right]
\end{aligned}
$$

where $d_{1}, d_{2}$, and $d_{3}$ are dimensionless model parameters.

\subsection{Summary}

In this section we summarize the initial/boundary-value problem and constitutive relations that have been described in the previous sections. 


\subsubsection{Strong form initial/boundary-value problem}

The strong form of the initial/boundary-value problem can be stated as

$$
(\mathbf{S})\left\{\begin{array}{rlrl}
\operatorname{Div}\left(2 \mathbf{F}\left(g(c) \frac{\partial W^{+}}{\partial \mathbf{C}}+\frac{\partial W^{-}}{\partial \mathbf{C}}\right)\right)+\mathbf{B} & =\rho_{0} \ddot{\mathbf{U}} & & \text { on } \left.\quad \Omega_{0} \times\right] 0, T[ \\
\frac{2 \ell_{0}}{\mathcal{G}_{c}^{0}}\left(\beta_{e} g^{\prime} \mathcal{H}+\beta_{p} g_{p}^{\prime}\left\langle W_{p}-W_{0}\right\rangle\right)+c-4 \ell_{0}^{2} c, \mathbf{X} \cdot c_{, \mathbf{X}} & =1 & & \text { on } \left.\quad \Omega_{0} \times\right] 0, T[ \\
U_{i} & =G_{i} & & \text { on } \left.\quad \partial \Omega_{0, G_{i}} \times\right] 0, T[ \\
T_{i} & =H_{i} & & \text { on } \left.\quad \partial \Omega_{0, H_{i}} \times\right] 0, T[ \\
c_{, \mathbf{X}} \cdot \mathbf{N} & =0 & & \text { on } \left.\quad \partial \Omega_{0} \times\right] 0, T[ \\
\mathbf{U}(\mathbf{X}, 0) & =\mathbf{U}_{0}(\mathbf{X}) & \mathbf{X} \in \Omega_{0} \\
\dot{\mathbf{U}}(\mathbf{X}, 0) & =\mathbf{V}_{0}(\mathbf{X}) & \mathbf{X} \in \Omega_{0}
\end{array}\right.
$$

where $G_{i}(\mathbf{X}, t)$ are prescribe displacement boundary conditions on $\partial \Omega_{0, G_{i}}, H_{i}(\mathbf{X}, t)$ are prescribed traction boundary conditions on $\partial \Omega_{0, H_{i}}, \mathbf{T}=\mathbf{P} \cdot \mathbf{N}$ is the Piola-Kirchhoff traction vector, $\mathbf{U}_{0}$ is an initial displacement, and $\mathbf{V}_{0}$ is an initial velocity. These equations of motion can be solved to find both the displacement field $\mathbf{U}(\mathbf{X}, t)$ and phase-field $c(\mathbf{X}, t)$.

\subsubsection{Damaged hyperelastic constitutive model}

The damaged elastic constitutive model in the reference configuration.

Positive, negative and damaged elastic strain energy density functions

$$
\begin{aligned}
& U\left(J^{e}\right)=\frac{1}{2} \kappa\left[\frac{1}{2}\left(J^{e 2}-1\right)-\ln J^{e}\right] \\
& \bar{W}\left(\overline{\mathbf{C}}, \mathbf{C}^{p}\right)=\frac{1}{2} \mu\left(\overline{\mathbf{C}}: \mathbf{C}^{p-1}-3\right) \\
& W^{+}\left(J^{e}, \overline{\mathbf{C}}, \mathbf{C}^{p}\right)= \begin{cases}U\left(J^{e}\right)+\bar{W}\left(\overline{\mathbf{C}}, \mathbf{C}^{p}\right) & J^{e} \geq 1 \\
\bar{W}\left(\overline{\mathbf{C}}, \mathbf{C}^{p}\right) & J^{e}<1\end{cases} \\
& W^{-}\left(J^{e}\right) \quad= \begin{cases}0 & J^{e} \geq 1 \\
U\left(J^{e}\right) & J^{e}<1\end{cases} \\
& W\left(J^{e}, \overline{\mathbf{C}}, \mathbf{C}^{p}\right)=g(c) W^{+}\left(J^{e}, \overline{\mathbf{C}}, \mathbf{C}^{p}\right)+W^{-}\left(J^{e}\right)
\end{aligned}
$$

Positive, negative and damaged second Piola-Kirchhoff stress tensors 


$$
\begin{aligned}
& \mathbf{S}^{+}= \begin{cases}J^{e} U^{\prime}\left(J^{e}\right) \mathbf{C}^{-1}+\mu J^{e-2 / 3}\left[\mathbf{C}^{p-1}-\frac{1}{3}\left(\mathbf{C}: \mathbf{C}^{p-1}\right) \mathbf{C}^{-1}\right] & J^{e} \geq 1 \\
\mu J^{e-2 / 3}\left[\mathbf{C}^{p-1}-\frac{1}{3}\left(\mathbf{C}: \mathbf{C}^{p-1}\right) \mathbf{C}^{-1}\right] & J^{e}<1\end{cases} \\
& \mathbf{S}^{-}= \begin{cases}\mathbf{0} & J^{e} \geq 1 \\
J^{e} U^{\prime}\left(J^{e}\right) \mathbf{C}^{-1} & J^{e}<1\end{cases} \\
& \mathbf{S}=g(c) \mathbf{S}^{+}+\mathbf{S}^{-}
\end{aligned}
$$

The Cauchy stress obtained by a standard push-forward of the second Piola-Kirchhoff stress

$$
\begin{aligned}
& \boldsymbol{\sigma}^{+}= \begin{cases}U^{\prime}\left(J^{e}\right) \mathbf{I}+J^{e-1} \mu \operatorname{dev}\left[\overline{\mathbf{b}}^{e}\right] & J^{e} \geq 1 \\
J^{e-1} \mu \operatorname{dev}\left[\overline{\mathbf{b}}^{e}\right] & J^{e}<1\end{cases} \\
& \boldsymbol{\sigma}^{-}= \begin{cases}\mathbf{0} & J^{e} \geq 1 \\
U^{\prime}\left(J^{e}\right) \mathbf{I} & J^{e}<1\end{cases} \\
& \boldsymbol{\sigma}=g(c) \boldsymbol{\sigma}^{+}+\boldsymbol{\sigma}^{-}
\end{aligned}
$$

\subsubsection{Elastoplastic constitutive model}

A finite deformation rate-independent elastoplastic constitutive model based on $J_{2}$ flow theory with an associative flow rule is considered. The stress response is governed by the damage elastic constitutive model from Section 3.4.2.

Elastic-plastic strain decomposition:

Isochoric assumption:

Constitutive model (see Section 3.4.2):

Mises-Huber yield function:

Flow rule:

$$
\mathbf{F}=\mathbf{F}^{e} \mathbf{F}^{p}
$$

$$
\begin{aligned}
\operatorname{det}\left[\mathbf{F}^{p}\right] & =1 \\
J^{e}=J & =\operatorname{det}[\mathbf{F}]
\end{aligned}
$$

$$
\boldsymbol{\sigma}=g(c) \boldsymbol{\sigma}^{+}+\boldsymbol{\sigma}^{-}, \quad \boldsymbol{\tau}=J^{e} \boldsymbol{\sigma}
$$

$$
f=\|\mathbf{s}\|-g_{p}(c) \sqrt{\frac{2}{3}} k(\alpha), \quad \mathbf{s}=\operatorname{dev} \boldsymbol{\tau}
$$

$$
\begin{aligned}
\operatorname{dev}\left[L_{v} \mathbf{b}^{e}\right] & =-\frac{2}{3} \dot{\gamma} \operatorname{tr}\left[\mathbf{b}^{e}\right] \mathbf{n}, \quad \mathbf{n}=\frac{\mathbf{s}}{\|\mathbf{s}\|} \\
\operatorname{det}\left[\mathbf{b}^{e}\right] & =J^{2}
\end{aligned}
$$


Hardening law: $\quad \dot{\alpha}=\sqrt{\frac{2}{3}} \dot{\gamma}$

Kuhn-Tucker loading/unloading conditions: $\quad f \leq 0, \quad \dot{\gamma} \geq 0, \quad f \dot{\gamma}=0$

\subsubsection{Effective plastic work}

The effective plastic work is calculated from the following rate equations:

No triaxiality:

With triaxiality:

$$
\dot{W}_{p}=\dot{\gamma}\|\mathbf{s}\|
$$

$$
\dot{W}_{p}=\dot{\gamma} \frac{\|\mathbf{s}\|}{\varphi}
$$

$$
\varphi=d_{1}+d_{2} \exp \left[d_{3} \frac{\tau_{m}}{\|\mathbf{s}\|}\right]
$$$$
\tau_{m}=\frac{\operatorname{tr}[\boldsymbol{\tau}]}{3}
$$

\section{Finite element formulation}

In this section we describe the discretization of the formulation developed in Section 3. We formulate the spatial discretization by means of the Galerkin method. The temporal discretization of the governing equations is achieved through an adaptation of the generalized- $\alpha$ method using a staggered scheme to solve for displacement and phase-field increments independently (see [8] for details). The temporal discretization of the elasto-plastic constitutive model is achieved via a backward Euler method that leads to an elastic predictor, plastic corrector algorithm.

The Galerkin formulation will follow from the weak form problem statement in the usual way. We let $\boldsymbol{g}$ be the given displacement boundary conditions on $\partial \Omega_{\boldsymbol{g}}, \boldsymbol{h}$ be the given traction boundary conditions on $\partial \Omega_{\boldsymbol{h}}, \boldsymbol{u}_{0}$ and $\dot{\boldsymbol{u}}_{0}$ be the initial displacement and velocity respectively, and $c_{0}$ be the initial phase-field. The trial solution spaces are

$$
\begin{aligned}
\mathcal{S}_{t} & =\left\{\boldsymbol{u}(t) \in H^{1}(\Omega)^{d} \mid u_{i}(t)=g_{i} \text { on } \partial \Omega_{g_{i}}\right\} \\
\widetilde{\mathcal{S}}_{t} & =\left\{c(t) \in H^{1}(\Omega)\right\}
\end{aligned}
$$

and the weighting function spaces are

$$
\mathcal{V}=\left\{\boldsymbol{w} \in H^{1}(\Omega)^{d} \mid w_{i}(t)=0 \text { on } \partial \Omega_{g_{i}}\right\}
$$




$$
\widetilde{\mathcal{V}}=\left\{q \in H^{1}(\Omega)\right\}
$$

The weak form can now be stated in the current configutation as:

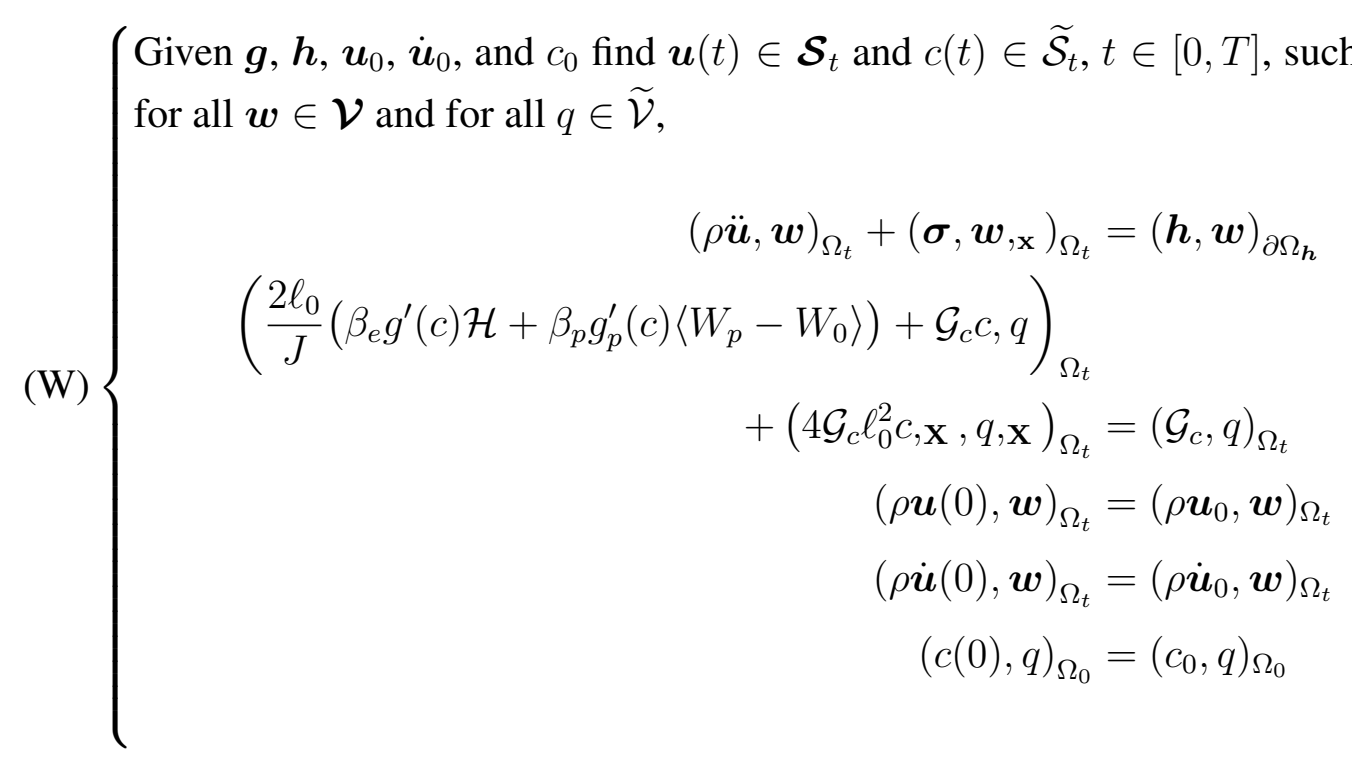

where $\mathcal{G}_{c}=\mathcal{G}_{c}^{0} / J$ is the critical fracture energy density in the current configuration.

Following the Galerkin method, we define the usual finite dimensional function spaces $\mathcal{S}_{t}^{h} \subset$ $\mathcal{S}_{t}, \widetilde{\mathcal{S}}_{t}^{h} \subset \widetilde{\mathcal{S}}_{t}, \mathcal{V}^{h} \subset \mathcal{V}$, and $\widetilde{\mathcal{V}}^{h} \subset \widetilde{\mathcal{V}}$ to arrive at the Galerkin form of the problem (see Hughes [22]), which is given by

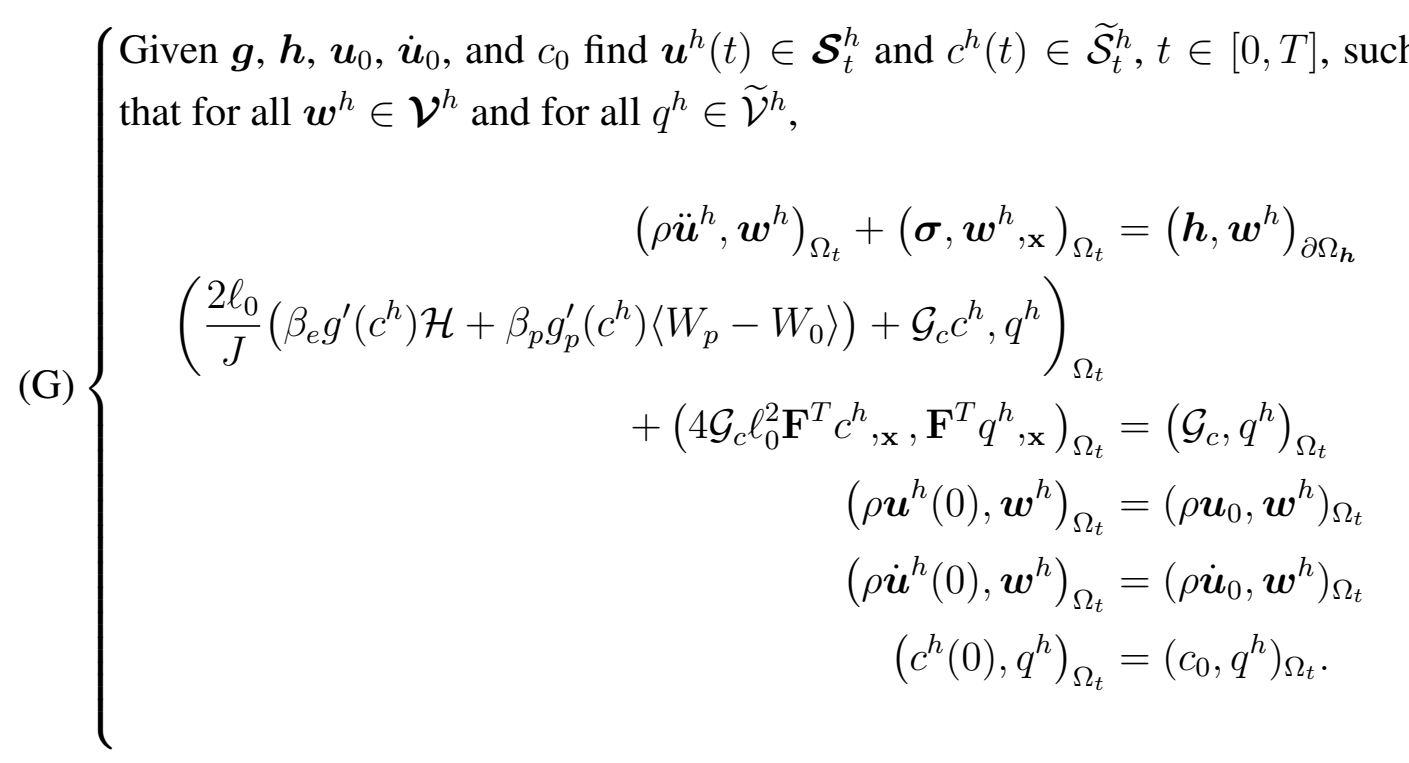

The explicit representation of the displacement and phase-field in terms of the basis functions and 
nodal variables is given by

$$
\begin{aligned}
\mathbf{u}^{h}(\mathbf{x}) & =\sum_{B=1}^{n_{\mathrm{b}}} N_{B}(\mathbf{x}) \mathbf{d}_{B} \\
c^{h}(\mathbf{x}) & =\sum_{B=1}^{n_{\mathrm{b}}} N_{B}(\mathbf{x}) \phi_{B}
\end{aligned}
$$

where $n_{\mathrm{b}}$ is the number of basis functions.

\subsection{Return-mapping}

During plastic flow the configuration is updated based on the elastoplastic constitutive theory summarized in Section 3.4.3. The updated configuration at time $t_{n+1}$ must satisfy the discrete form of the Karush-Kuhn-Tucker conditions

$$
f\left(\boldsymbol{\tau}_{n+1}, \alpha_{n+1}\right) \leq 0, \quad \Delta \gamma \geq 0, \quad \Delta \gamma f\left(\boldsymbol{\tau}_{n+1}, \alpha_{n+1}\right)=0
$$

where $\boldsymbol{\tau}_{n+1}=\operatorname{tr}\left[\boldsymbol{\tau}_{n}\right] \mathbf{I}+g(c) \mu \operatorname{dev}\left[\overline{\mathbf{b}}_{n+1}^{e}\right]$ and $\Delta \gamma$ is the algorithmic counterpart to $\dot{\gamma}$. For each time increment $\left[t_{n}, t_{n+1}\right]$ we assume a trial state based on a purely elastic deformation, i.e.

$$
\begin{aligned}
\overline{\boldsymbol{f}}_{n+1} & =\operatorname{det}\left[\boldsymbol{f}_{n+1}\right]^{-1 / 3} \boldsymbol{f}_{n+1} \\
\overline{\mathbf{b}}_{n+1}^{\text {thial }} & =\overline{\boldsymbol{f}}_{n+1} \overline{\mathbf{b}}_{n}^{e} \overline{\boldsymbol{f}}_{n+1}^{T} \\
\alpha_{n+1}^{\text {trial }} & =\alpha_{n}
\end{aligned}
$$

where

$$
\boldsymbol{f}_{n+1}=\mathbf{I}+\nabla_{x_{n}} \mathbf{u}_{n}
$$

is the relative deformation gradient between the configurations at time $t_{n}$ and $t_{n+1}$. If the trial state satisfies the discrete KKT conditions then it is accepted as the updated configuration. If the discrete KKT conditions are not satisfied then the trial state is inadmissible and the configuration must be updated according to the discrete form of the flow rule and hardening law

$$
\begin{aligned}
\operatorname{dev}\left[\overline{\mathbf{b}}_{n+1}^{e}\right] & =\operatorname{dev}\left[\overline{\mathbf{b}}_{n+1}^{e \text { trial }}\right]-\frac{2}{3} \Delta \gamma \operatorname{tr}\left[\overline{\mathbf{b}}_{n+1}^{e}\right] \mathbf{n}_{n+1} \\
\alpha_{n+1} & =\alpha_{n}+\sqrt{\frac{2}{3}} \Delta \gamma
\end{aligned}
$$


This update leads to the return-mapping algorithm outlined in detail in Appendix B and summarized in the following section.

We point out that this algorithm deviates from the algorithm presented in [43] in two ways. First, we have included the elastic and plastic degradation functions from the theory presented in Section 3. Second, we have modified the update of the intermediate configuration (Step 6) to maintain the correct determinant of $\overline{\mathbf{b}}^{e}$. This update effects only the trace of $\overline{\mathbf{b}}^{e}$ and has no effect on the stress computations but is necessary to compute the correct strain energy. More details and further justification are provided in Appendix B.

\subsubsection{The return-mapping algorithm}

The steps of the return-mapping algorithm are summarized in this section. This algorithm follows the algorithm presented by Simo and Hughes [43] with modifications to include the degradation functions and correct the update of the intermediate configuration. The modifications are indicated by the boxed lines.

1. Update the current configuration

$$
\begin{aligned}
& \phi_{n+1}(\mathbf{X})=\phi_{n}(\mathbf{X})+\mathbf{u}_{n}\left(\phi_{n}(\mathbf{X})\right) \\
& \boldsymbol{f}_{n+1}=\mathbf{I}+\nabla_{x_{n}} \mathbf{u}_{n} \\
& \mathbf{F}_{n+1}=\boldsymbol{f}_{n+1} \mathbf{F}_{n}
\end{aligned}
$$

2. Compute the damage/elastic predictor

$$
\begin{aligned}
& \overline{\boldsymbol{f}}_{n+1}=\operatorname{det}\left[\boldsymbol{f}_{n+1}\right]^{-1 / 3} \boldsymbol{f}_{n+1} \\
& \overline{\mathbf{b}}_{n+1}^{e \text { trial }}=\overline{\boldsymbol{f}}_{n+1} \overline{\mathbf{b}}_{n}^{e} \overline{\boldsymbol{f}}_{n+1}^{T} \\
& \mathbf{s}_{n+1}^{\text {trial }}=g(c) \mu \operatorname{dev}\left[\overline{\mathbf{b}}_{n+1}^{\text {etrial }}\right]
\end{aligned}
$$

3. Check for plastic loading

$$
\begin{aligned}
& f_{n+1}^{\text {trial }}=\left\|\mathbf{s}_{n+1}^{\text {trial }}\right\|-g_{p}\left(c_{n}\right) \sqrt{\frac{2}{3}} k\left(\alpha_{n}\right) \\
& \text { IF } f_{n+1}^{\text {trial }} \leq 0 \text { THEN } \\
& \text { Set }(\cdot)_{n+1}=(\cdot)_{n+1}^{\text {trial }}, \text { and EXIT }
\end{aligned}
$$

ELSE

$$
\text { Go to } 4 \text { (return-mapping) }
$$

ENDIF 
4. Return-mapping

$\bar{I}_{n+1}^{e \text { trial }}=\frac{1}{3} \operatorname{tr}\left[\overline{\mathbf{b}}_{n+1}^{e \text { trial }}\right]$

$\bar{\mu}=g(c) \mu \bar{I}_{n+1}^{e \text { trial }}$

Solve: $\hat{f}(\Delta \gamma)=\left\|\mathbf{s}_{n+1}^{\text {trial }}\right\|-g_{p}\left(c_{n}\right) \sqrt{\frac{2}{3}} k\left(\alpha_{n}+\sqrt{\frac{2}{3}} \Delta \gamma\right)-2 \bar{\mu} \Delta \gamma=0$

$$
\begin{aligned}
& \mathbf{s}_{n+1}=\mathbf{s}_{n+1}^{\text {trial }}-2 \bar{\mu} \Delta \gamma \mathbf{n} \\
& \alpha_{n+1}=\alpha_{n}+\sqrt{\frac{2}{3}} \Delta \gamma
\end{aligned}
$$

5. Update the Kirchhoff stress

$$
\begin{aligned}
& J_{n+1}=\operatorname{det}\left[\mathbf{F}_{n+1}\right] \\
& p_{n+1}= \begin{cases}g(c) U^{\prime}\left(J_{n+1}\right) & J_{n+1} \geq 1 \\
U^{\prime}\left(J_{n+1}\right) & J_{n+1}<1\end{cases} \\
& \boldsymbol{\tau}_{n+1}=J_{n+1} p_{n+1} \mathbf{I}+\mathbf{s}_{n+1}
\end{aligned}
$$

6. Update the intermediate configuration (corrects error in algorithm presented in [43])

$$
\begin{aligned}
& \operatorname{dev}\left[\overline{\mathbf{b}}_{n+1}^{e}\right]=\frac{\mathbf{s}_{n+1}}{g(c) \mu} \\
& \text { Solve (to find } \left.\bar{I}_{n+1}^{e}\right): \operatorname{det}\left[\operatorname{dev}\left[\overline{\mathbf{b}}_{n+1}^{e}\right]+\bar{I}_{n+1}^{e} \mathbf{I}\right]=1 \\
& \overline{\mathbf{b}}_{n+1}^{e}=\operatorname{dev}\left[\overline{\mathbf{b}}_{n+1}^{e}\right]+\bar{I}_{n+1}^{e} \mathbf{I}
\end{aligned}
$$

\section{Numerical Results}

In the previous sections we have introduced a number of modifications to the phase-field theory to facilitate its application to ductile materials. These modifications include:

1. The introduction of a cubic degradation function that more accurately represents the linear stress-strain response prior to crack initiation/nucleation (Section 2.2.1)

2. The use of a plastic work threshold in the phase-field equation (Section 2.3.1).

3. An additional degradation function that induces plastic softening (Section 3.2). 
4. A dependence of the effective plastic work measure on the state of stress triaxiality (Section 3.3).

In the following sections we present results for a number of numerical experiments that demonstrate the effects of these modifications. The first is a series of computations for a uniaxial stress state that establish the stress-strain response of the model. The second is a computation on a thin, flat, and symmetrically notched specimen that is loaded in tension. This example allows us to explore the stress-strain behavior of a three-dimensional geometry. We also present an adaptive time stepping scheme in conjunction with this example that provides a mechanism for adjusting the dynamic time step size based on the evolution of the phase-field. The third example represents a contemporary problem that is of interest to the defense industry. In this example we simulate a large steel plate subjected to a blast load. This example demonstrates that phase-field models have the potential to predict complex failure behavior of three-dimensional structures under severe load conditions.

\subsection{Uniaxial constitutive response}

In this section we present an analysis of the effects of modifications 1-3 on the constitutive response of the proposed model in a simple one-dimensional setting (we examine the effect of modification 4 in Section 5.2). This analysis will help to develop an intuitive understanding of how the modifications we have introduced effect the response. To simplify the analysis, the response will be computed on a single unit cube volumetric element under a state of uniaxial stress. Furthermore, we choose a length scale, $\ell_{0}$, that is larger than the element size. This creates a homogeneous response in $c$ where all derivatives of $c$ are equal to zero. We will also ignore inertial and body force terms. The model parameters are given in Table 1 and yield $\sigma_{c}=500 \mathrm{MPa}$.

Table 1: Model parameters for the uniaxial stress computations reported in this section.

\begin{tabular}{rl}
\hline Young's modulus & $68.8 \mathrm{GPa}$ \\
Poisson's ratio & 0.33 \\
Yield stress & $320 \mathrm{MPa}$ \\
Critical fracture energy & $138 \times 10^{6} \mathrm{~J} / \mathrm{m}^{2}$ \\
$\ell_{0}$ & $2 \mathrm{~m}$ \\
\hline
\end{tabular}

We first examine the case where $g_{p}=1$ (there is no plastic softening) $g$ is taken to be the standard quadratic degradation function, and $W_{0}=0$. Two variations of this case are shown in 
Figure 5 and are compared to the standard $J_{2}$-plasticity response with linear isotropic hardening, which we refer to as the "No fracture" case. For the first variant we set $\beta_{e}=1$ and $\beta_{p}=0$ so that only the elastic strain energy contributes directly to the evolution of the phase-field. For the second variant of this case we set $\beta_{e}=\beta_{p}=1$ to show the response when the effective plastic work is also included in the phase-field equation with $W_{0}=0$.
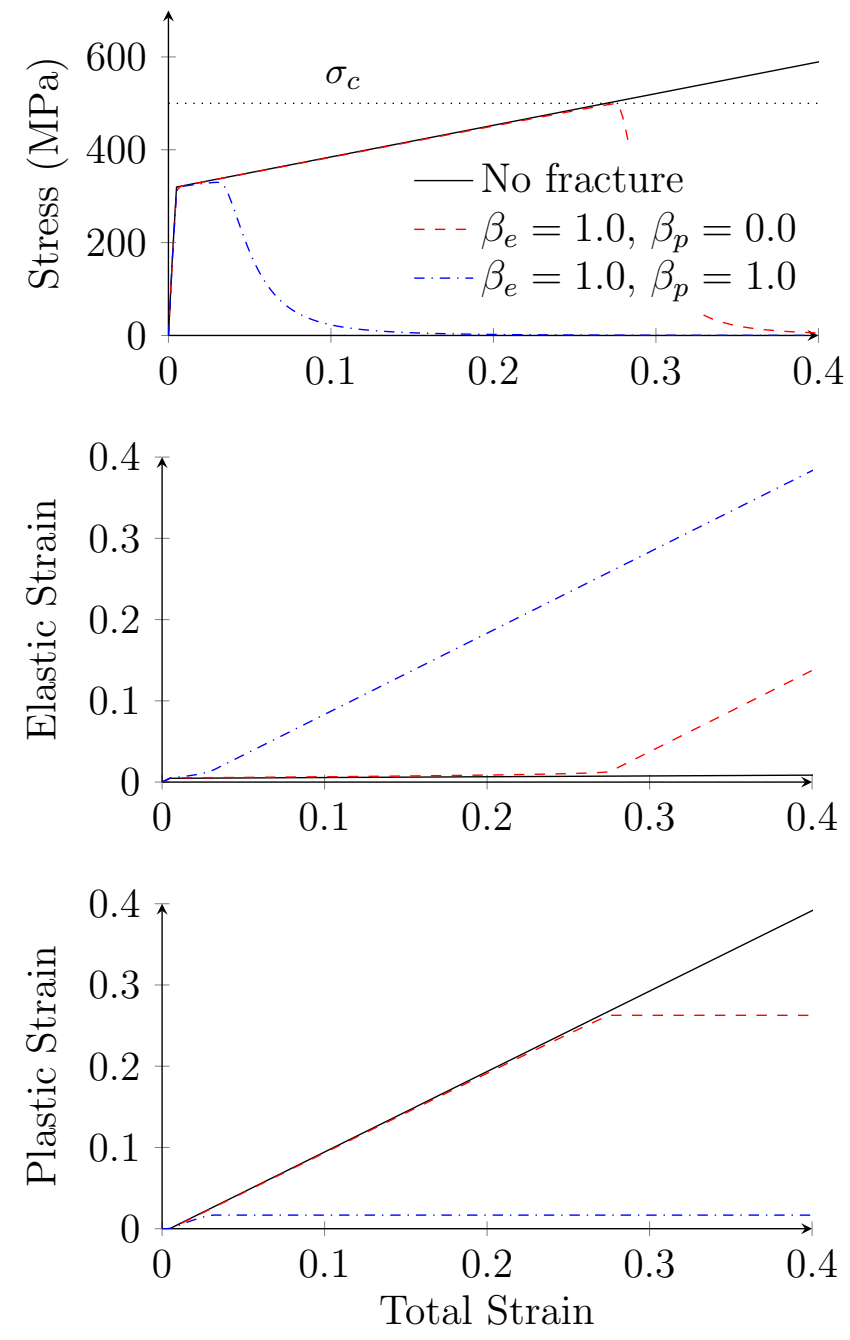

Figure 5: The stress-strain response for a simple $J_{2}$ plasticity model with linear hardening. The "No fracture" case is a simple elastic-plastic response with linear hardening and is shown for reference. When $\beta_{p}=0.0$ the stress increases until it hits the critical stress that is predicted by the brittle theory. When $\beta_{p}=1.0$ we see the response when the effective plastic work term is added to the history functional.

A number of observations can be made from the plots shown in Figure 5. First, when $\beta_{e}=1$ and $\beta_{p}=0$ the stress reaches the critical stress, labeled $\sigma_{c}$, that is calculated for the purely elastic 
case. This is expected since the driving term in the phase-field equation includes only contributions from the elastic strains. Second, setting $\beta_{p}=1$ reduces the critical stress and crack formation occurs at a much lower total strain. Third, the plots of elastic and plastic strains show that the plastic strain saturates at about the level of total strain where the ultimate stress is obtained. Beyond this point, the model exhibits damage elastic behavior. This is contrary to what is expected from ductile materials where very large plastic strains occur.

The nonphysical behavior of the strains shown in Figure 5 motivated the addition of the plastic degradation function to the yield surface. Adding the degradation function to the yield surface, as described in Section 3.2, changes the constitutive response to that shown in Figure 6. For this response, the quadratic degradation function was used for both $g$ and $g_{p}$. In this case the strain response is as expected: once yielding occurs the total strain is dominated by plastic strain. This happens because the plastic degradation function is shrinking the yield surface even as the elastic strength is being reduced. For isotropic hardening, as in the current case, there will be competition between the hardening effect from additional plastic strain and the softening due to the evolution of $c$ and $g_{p}(c)$. For the current response, this results in a reduction in strain hardening as shown by the reduced slope of the curve after yielding. We also see in Figure 6 that the initial yield stress is lower compared to the no fracture case. This is a result of the reduction in $c$, and consequently in $g_{p}$, prior to reaching the elastic critical stress as is shown in Figure 7.

For the constitutive response shown in Figure 8 we have used the cubic degradation function (14) with $m=10^{-4}$ for both $g$ and $g_{p}$. For this case we have adjusted the material parameters such that the critical stress in the elastic case is the same as it would be if the quadratic degradation function where used (compare Figure 7 to Figure 2). We can see that the yield stress in this case is the same in the damaged and undamaged cases because the cubic degradation function delays damage accumulation due to elastic deformation until just before the critical stress is reached. Once the critical stress is reached, the more rapid decrease of the cubic $g_{p}$ with increased elastic strain (when compared to the quadratic $g_{p}$ ) causes softening as the yield surface contracts due to $g_{p}$ out pacing the expansion caused by the evolution of the hardening parameter $\alpha$.

\subsection{Tensile Test}

In this section we simulate crack initiation and growth in a symmetrically notched thin rectangular specimen, shown in Figure 9, which is loaded in tension. The notched configuration causes a nonuniform triaxiality state prior to necking on the cross section bisecting the specimen between the notches. This allows us to investigate the effect of the modification to the effective plastic work computation presented in Section 3.3. 

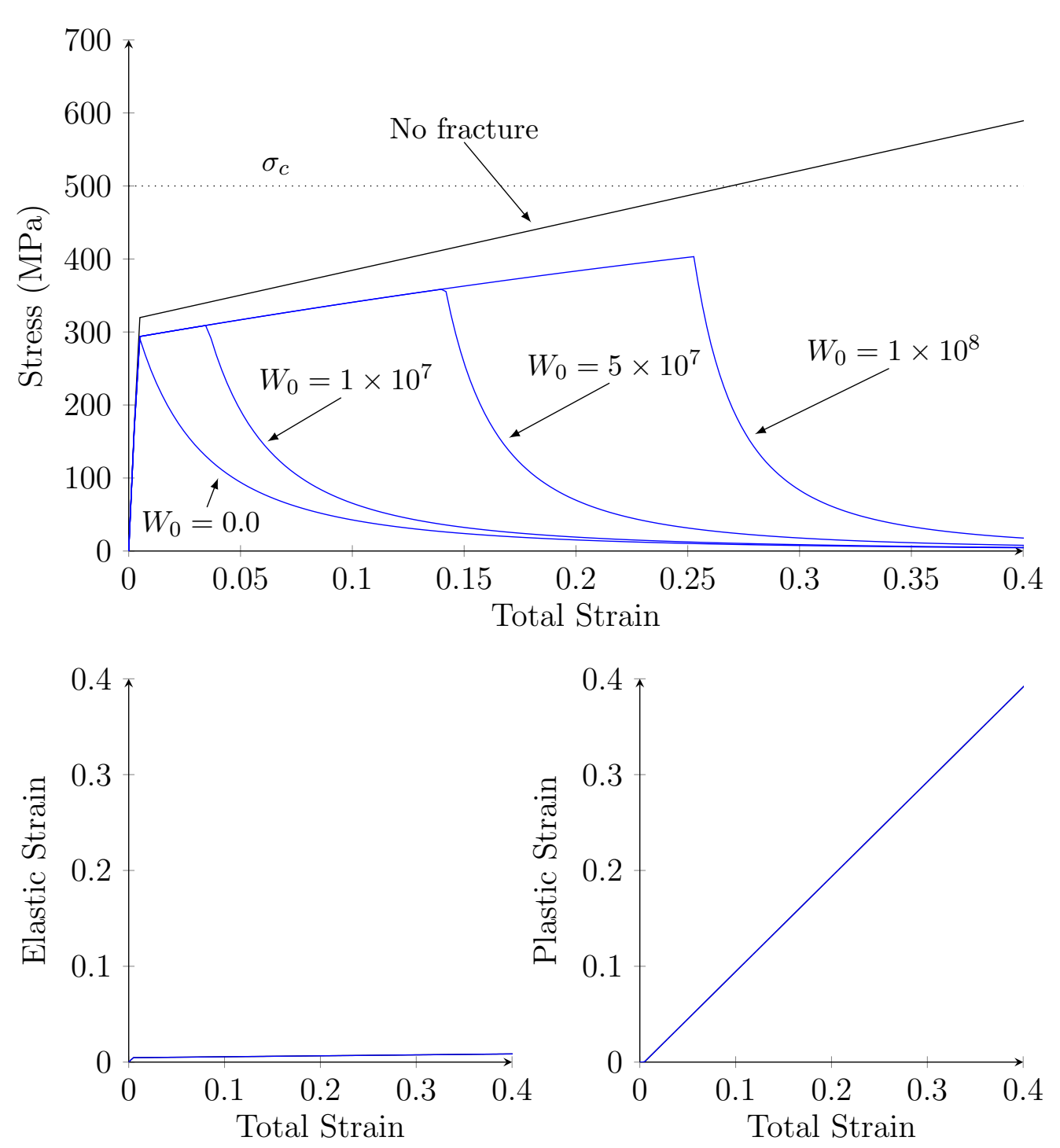

Figure 6: Stress-strain constitutive response using the quadratic degradation function for both $g$ and $g_{p}$ for several different values for the plastic work threshold $W_{0}$. The initial yield stress is reduced due to accumulated damage caused by elastic strains prior to yielding. Strain hardening is reduced due to the contraction of the yield surface induced by $g_{p}$. The elastic and plastic strain curves follow the "No fracture" case for all values of $W_{0}$.

The computations were performed on a multi-patch quadratic NURBS isogeometric spatial discretization (see Hughes et al. [23]). The Bézier element representation of the NURBS patches is shown in Figure 10. This discretization is refined locally using a hierarchical refinement scheme as described in Schillinger et al. [40] and Scott et al. [41]. Using hierarchical NURBS allows us to represent the circular notches exactly in the analysis model. This is an important consideration 

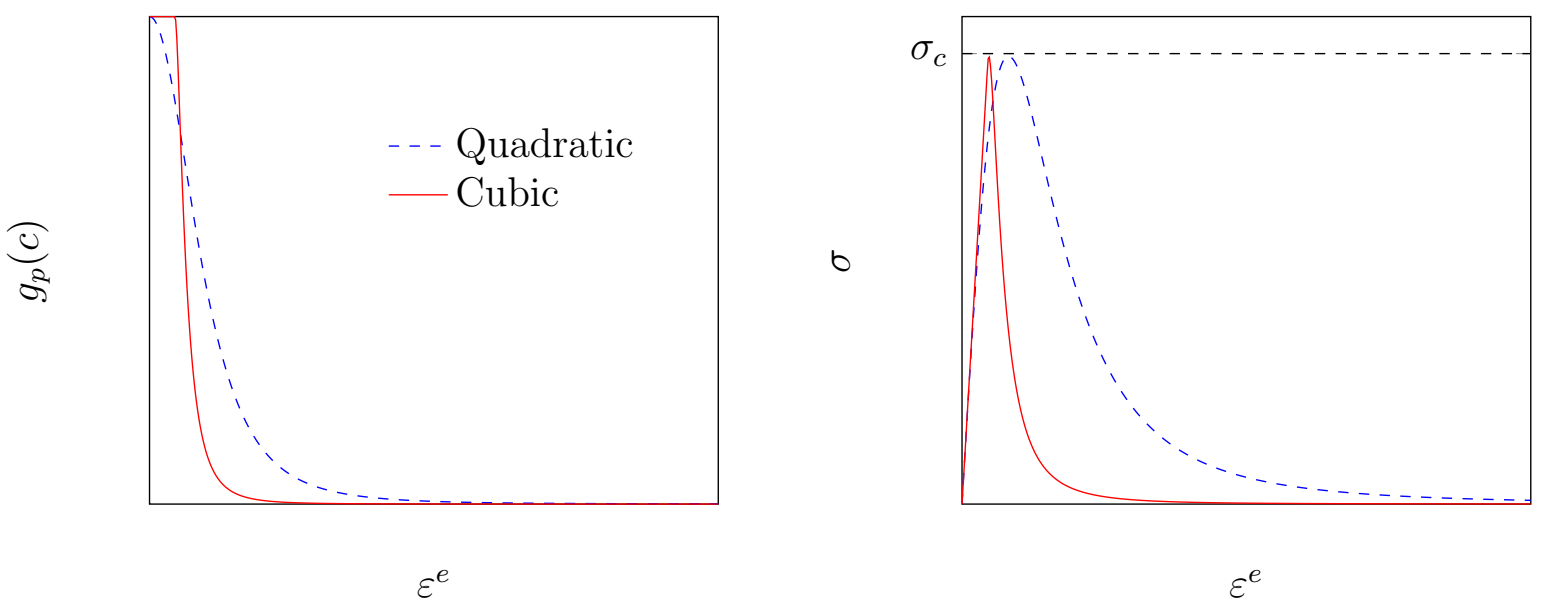

Figure 7: The quadratic and cubic degradation function (left) and the respective stress-strain plots for a uniaxial homogeneous constitutive response (right). The length scale parameter has been adjusted for the response from the cubic degradation function so that the critical stress is the same for both cases. Compare the stress-strain response from Figure 2.

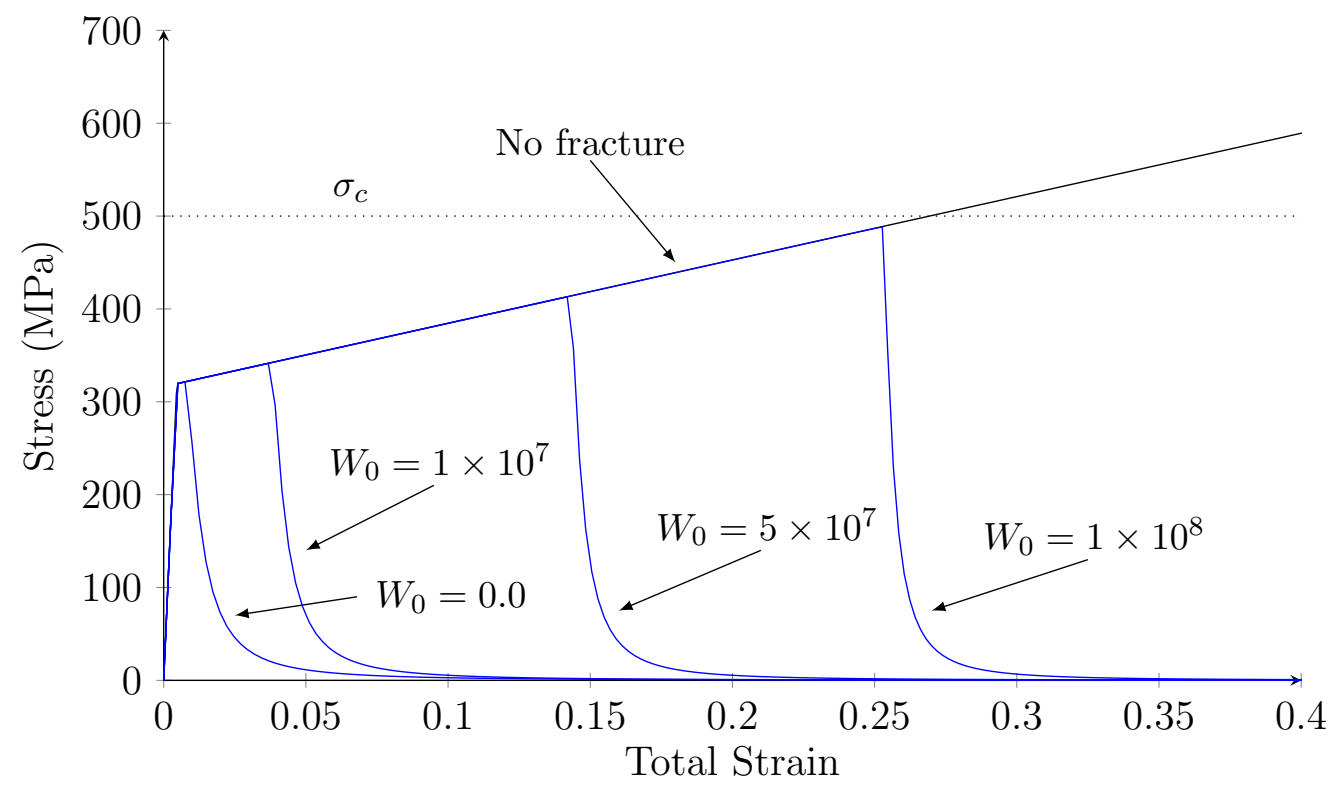

Figure 8: Stress-strain constitutive response using the cubic degradation function for both $g_{e}$ and $g_{p}$ with several different values for the plastic work threshold $W_{0}$. The cubic degradation delays the accumulation of damage until either the critical stress or plastic work threshold is achieved so that the original yield stress is maintained. The elastic and plastic strain curves (not shown) follow the "No fracture" case for all values of $W_{0}$.

for fracture problems because the stress intensity factors that drive crack growth are influenced by the geometry of the problem. Furthermore, the resolution of the discretization and the higher 


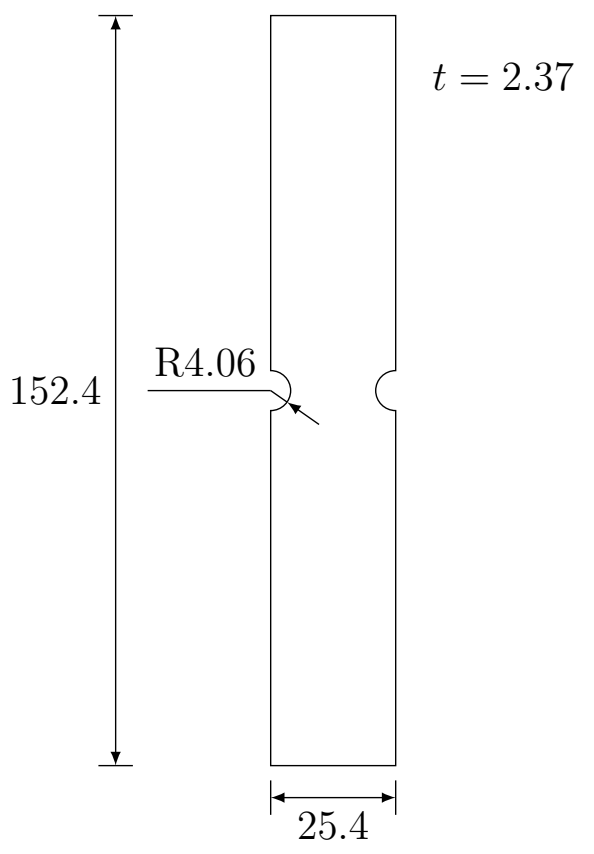

Figure 9: The geometry and associated dimensions (in $\mathrm{mm}$ ) used for the tensile test. A thin rectangular specimen was notched at the midpoint to localize stresses and initiate yielding and crack growth.

polynomial degree of the basis reduces the effect of volumetric locking during plastic deformation and no special treatment of the volumetric term is used in these calculations.

The material parameters used for the numerical simulations are shown in Table 2. The parameters $E, \nu, \rho, \sigma_{y}$, and $k$ are fixed for all cases presented in the following. In choosing the remaining parameters we seek to keep the critical stress, $\sigma_{c}$, which depends on the choice of degradation function, and the length scale parameter, $\ell_{0}$, which effects the plastic response, the same. Under these constraints the only remaining parameter that can be changed is $\mathcal{G}_{c}^{0}$. For these simulations we have chosen a value for $\mathcal{G}_{c}^{0}$ for each case of the degradation function that yields the same critical stress value. This implies that the energy dissipated by the creation of the crack surfaces will be different between the cases using the quadratic and cubic degradation functions. However, for ductile materials the energy dissipated by plastic deformation is typically much larger than the energy required to create new crack surfaces and by keeping $\ell_{0}$ constant the plastic deformation is comparable for the two degradation functions.

The deformation is displacement controlled with the top and bottom surfaces being displaced in opposite directions at a rate of $4.2 \times 10^{-6} \mathrm{~m} / \mathrm{s}$, which maintains a quasi-static loading condition. The computation, however, was fully dynamic and the staggered solution scheme described in [8] was used at each time increment with the momentum equation being solved implicitly. 
Table 2: Material properties for the notched specimen tensile test.

\begin{tabular}{ll}
\hline Young's modulus $(E)$ & $68.8 \mathrm{GPa}$ \\
Poisson's ratio $(\nu)$ & 0.33 \\
Mass density $(\rho)$ & $2700 \mathrm{~kg} / \mathrm{m}^{3}$ \\
Yield stress $\left(\sigma_{y}\right)$ & $320 \mathrm{MPa}$ \\
Hardening modulus $(k)$ & $688 \mathrm{MPa}$ \\
Critical fracture energy $\left(\mathcal{G}_{c}^{0}\right)-$ quadratic & $60 \times 10^{3} \mathrm{~J} / \mathrm{m}^{2}$ \\
Critical fracture energy $\left(\mathcal{G}_{c}^{0}\right)-$ cubic & $18.06 \times 10^{3} \mathrm{~J} / \mathrm{m}^{2}$ \\
Length scale $\left(\ell_{0}\right)$ & $3.226 \times 10^{-4} \mathrm{~m}$ \\
Triaxiality parameter $1\left(d_{1}\right)$ & 0.1 \\
Triaxiality parameter $2\left(d_{2}\right)$ & 3.8 \\
Triaxiality parameter $3\left(d_{3}\right)$ & -1.8 \\
\hline
\end{tabular}

\subsubsection{Adaptive Time Stepping}

Because of the quasi-static loading rate there is a relatively long period of time over which the load is applied but no crack propagation occurs. During this time interval the inertial terms can be negligible, which allows time steps that are much larger than what is required to resolve dilatational waves. Once the crack initiates, however, the time step needs to be small enough to accurately capture the propagation of the crack. We propose here a heuristic adaptive time stepping scheme that efficiently handles the large difference in the time scales between the quasi-static loading stage and the crack propagation stage of this problem. This adaptive time stepping scheme adjusts the time step based on the change in the fracture energy between two consecutive time steps. The fracture energy is computed as

$$
\Psi_{n}^{c}=\int_{\Omega_{0}} \frac{\mathcal{G}_{c}^{0}}{4 \ell_{0}}\left[\left(c_{n}-1\right)^{2}+4 \ell_{0}^{2}\left|\left(c_{n}\right), \mathbf{x}\right|^{2}\right] d \Omega_{0}
$$

where the subscript $n$ refers to the time step number. The change in the fracture energy between two time steps acts as a surrogate measure for the crack tip velocity, which provides a way to determine when cracks are propagating. The scheme is summarized as follows:

1. Compute the change in fracture energy:

$$
\Delta \Psi_{n}^{c}=\Psi_{n}^{c}-\Psi_{n-1}^{c}
$$

2. Compute the effective change in the crack length by normalizing the change in fracture 

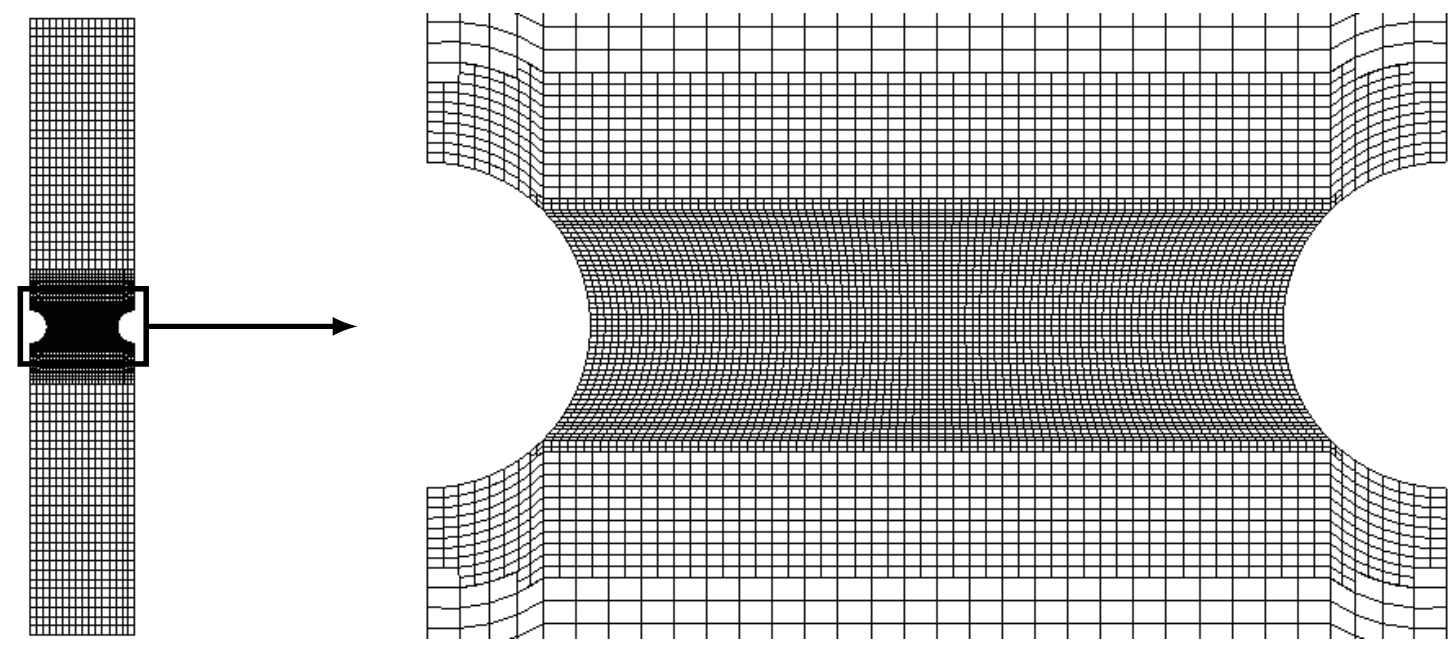

Figure 10: The Bézier representation of the NURBS discretization of the tensile test geometry. The basis functions are locally refined using hierarchical B-splines to maintain computational efficiency.

energy with respect to $\mathcal{G}_{c}^{0}$ :

$\Delta a_{n}^{\mathrm{eff}}=\frac{\Delta \Psi_{n}^{c}}{\mathcal{G}_{c}^{0}}$

3. Check to see if the time step should be reduced or increased based on a maximum and minimum tolerance for the ratio between $\Delta a_{n}^{\text {eff }}$ and $h_{\min }$. The tolerances are given as tol $\max$ and tol $_{\text {min. }}$. A safety parameter, $s_{a}$, is also specified to prevent large changes in the time step between consecutive time increments:

IF $\frac{\Delta a_{n}^{\text {eff }}}{h_{\min }}>$ tol $_{\max }$ THEN:

$$
\Delta t_{n+1}=\Delta t_{n}\left(\frac{\text { tol }_{\max }}{\Delta a^{\text {eff }} / h_{\min }}\right) \times s_{a}
$$

IF $\Delta t_{n+1}<\Delta t_{\text {min }}$ THEN

$$
\Delta t_{n+1}=\Delta t_{\min }
$$

END IF 


$$
\begin{gathered}
\text { ELSE IF } \frac{\Delta a_{n}^{\text {eff }}}{h_{\text {min }}}<\text { tol }_{\text {min }} \text { THEN } \\
\Delta t_{n+1}=\Delta t_{n}\left(\frac{\text { tol }_{\text {min }}}{\Delta a^{\text {eff }} / h_{\text {min }}}\right) \times s_{a} \\
\text { IF } \Delta t_{n+1}>2 \Delta t_{n} \text { THEN } \\
\Delta t_{n+1}=2 \Delta t_{n}
\end{gathered}
$$

END IF

IF $\Delta t_{n+1}>\Delta t_{\max }$ THEN

$$
\Delta t_{n+1}=\Delta t_{\max }
$$

END IF

\section{END IF}

The adaptive time stepping parameters used for the notched specimen example are given in Table 3.

Table 3: Parameters used to control the adaptive time stepping procedure for the notched specimen example.

\begin{tabular}{ll}
\hline tol $_{\max }$ & $10^{-4}$ \\
tol $_{\min }$ & tol $_{\max } / 2$ \\
$\Delta t_{\min }$ & $10^{-9} \mathrm{~s}$ \\
$\Delta t_{\max }$ & $0.5 \mathrm{~s}$ \\
$s$ & 0.9 \\
$h_{\min }$ & $1.5 \times 10^{-4} \mathrm{~m}$ \\
\hline
\end{tabular}

\subsubsection{Results}

Results were computed using both the quadratic and cubic degradation functions and unmodified and modified effective plastic work calculations. The computations were performed for several different values of the plastic work threshold, $W_{0}$. Plots showing the nominal stress vs. nominal strain for each case are presented in Figure 11 where the nominal stress is defined as the resultant force at the ends of the specimen divided by the undeformed cross-sectional area at the narrowest point in the notch and the nominal strain is defined as the total change in length of the specimen 
divided by the original length. Values of $W_{0}$ were first chosen for the computations without the modifications that include triaxiality effects. Then, for the cases with triaxiality included, values of $W_{0}$ where chosen so that the uniaxial constitutive response reaches the same maximum stress as the corresponding case without triaxiality effects. Values of $W_{0}$ were chosen in this way to facilitate comparison since the triaxiality modification decreases the effective plastic work. The plots in Figure 11 show qualitative agreement with the behavior seen for the one-dimensional response described in Section 5.1. That is, when the quadratic degradation function is used the yield stress is reduced but when using the cubic degradation function the response follows the no fracture case until crack nucleation. The effect of increasing the plastic work threshold also agrees with the uniaxial stress computations.

Contour plots showing the progression of the phase-field through the horizontal plane with minimum cross-section area are presented in Figures 12 and 13. These plot compare the results computed with and without triaxiality effects. For the plots shown in Figure 12 the quadratic degradation function was used, and the cubic degradation function was used for the plots shown in Figure 13. In both figures the effect of triaxiality is evident. Without triaxiality the crack nucleates on the surface at the notch. When triaxiality is included, the crack nucleates near the notch but not on the surface. This is the location of highest triaxiality as shown by Ghahremaninezhad and Ravi-Chandar [17].

Remark 5.1. It is well known that the state of stress in thin specimens depends on the thickness of the specimen, see for example [37]. Although a thorough investigation of the performance of the proposed model on specimens of different thickness is warranted it is beyond the scope of this paper and left for future efforts.

Remark 5.2. In the case of brittle fracture the energy dissipated by crack growth (creation of new surfaces) is much more important and the model parameters should be chosen to match the material behavior. Typically the critical stress and critical energy release rate are determined experimentally and the length scale parameter is chosen such that the stress-strain behavior fits the critical stress. This will result in different length scales being used for the quadratic and cubic degradation functions. For brittle fracture the choice of degradation function provides a means for tuning the the effective process zone around the crack tip. Further investigation is needed to better understand this behavior. 


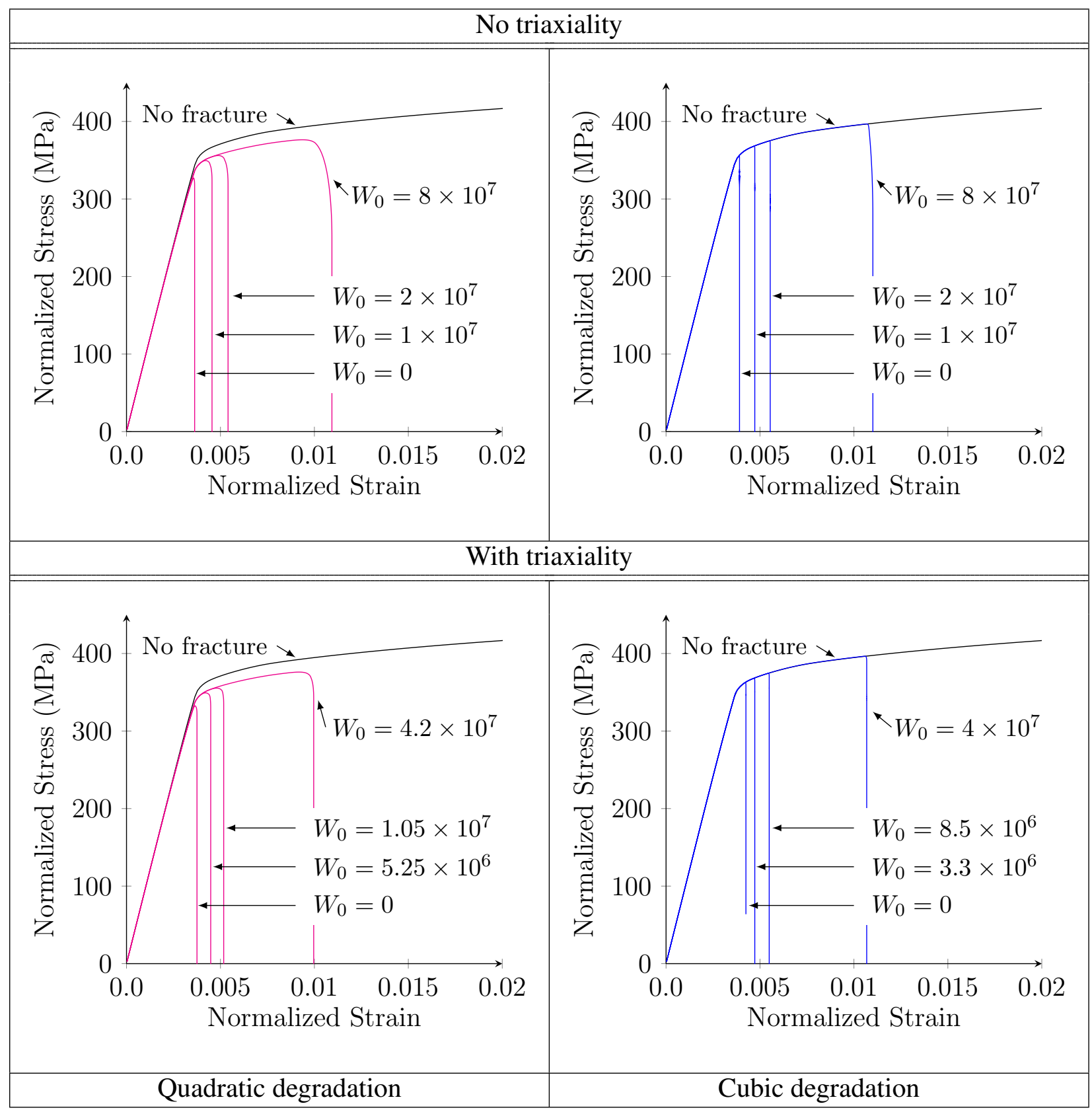

Figure 11: Normalized stress vs. normalized displacement curves for the tensile test results. The curve labeled "No fracture" is the response for standard $J_{2}$ plasticity with linear isotropic hardening. The top row shows the response with no triaxiality consideration and the bottom row shows the response when triaxiality is included in the effective plastic work computations. The right and left column show the difference when the quadratic and cubic degradation functions are used respectively. Values of $W_{0}$ where chosen for the calculations with triaxiality such that the uniaxial constitutive response reaches the same maximum stress as the corresponding case without triaxiality effects. 

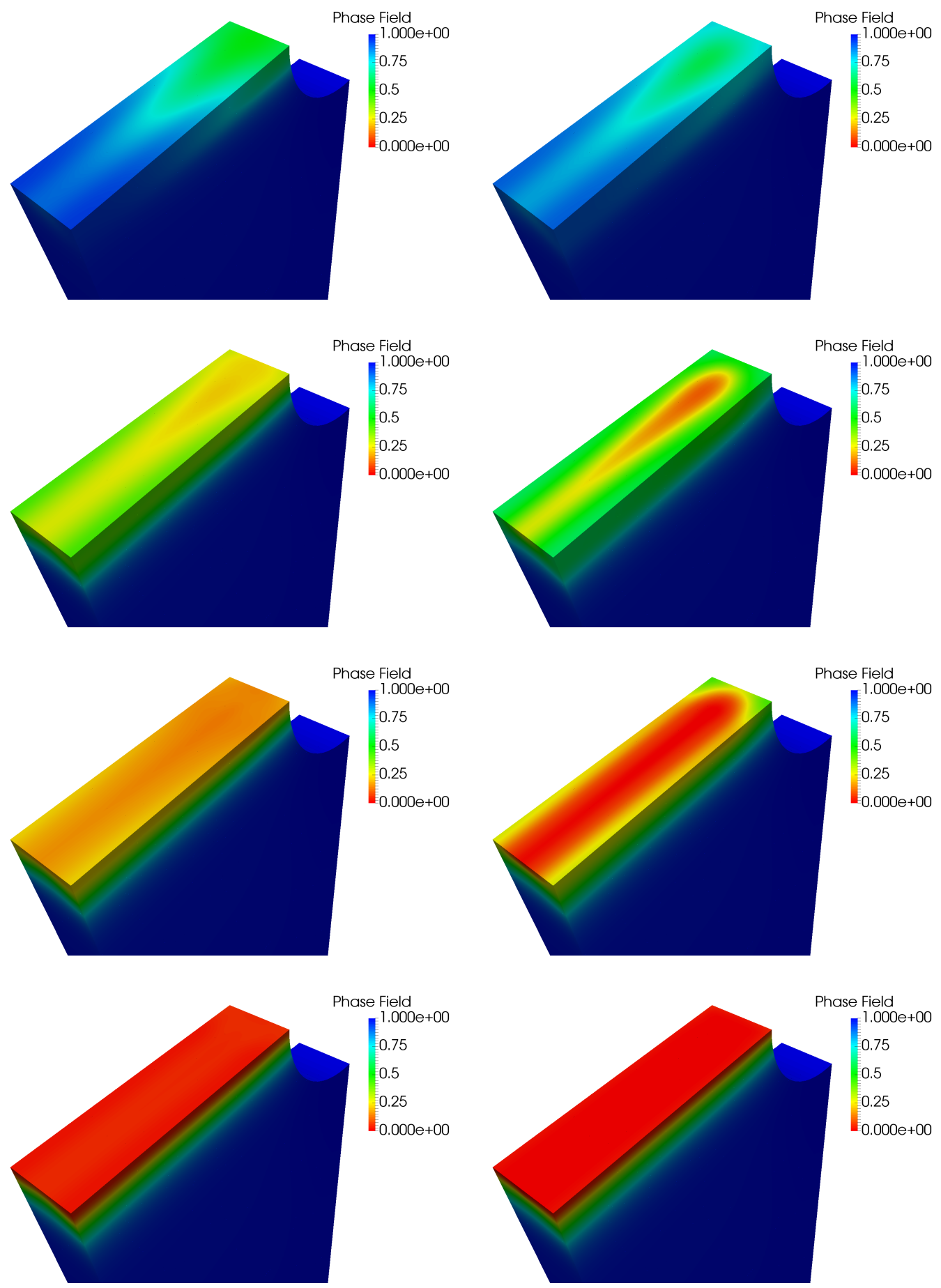

No triaxiality

With triaxiality

Figure 12: Contour plots of the phase-field as the crack progresses through a horizontal plane through the minimum cross-section area using the quadratic degradation function. The left column shows the results when triaxiality is not considered and the right column shows the results when triaxiality is included. 

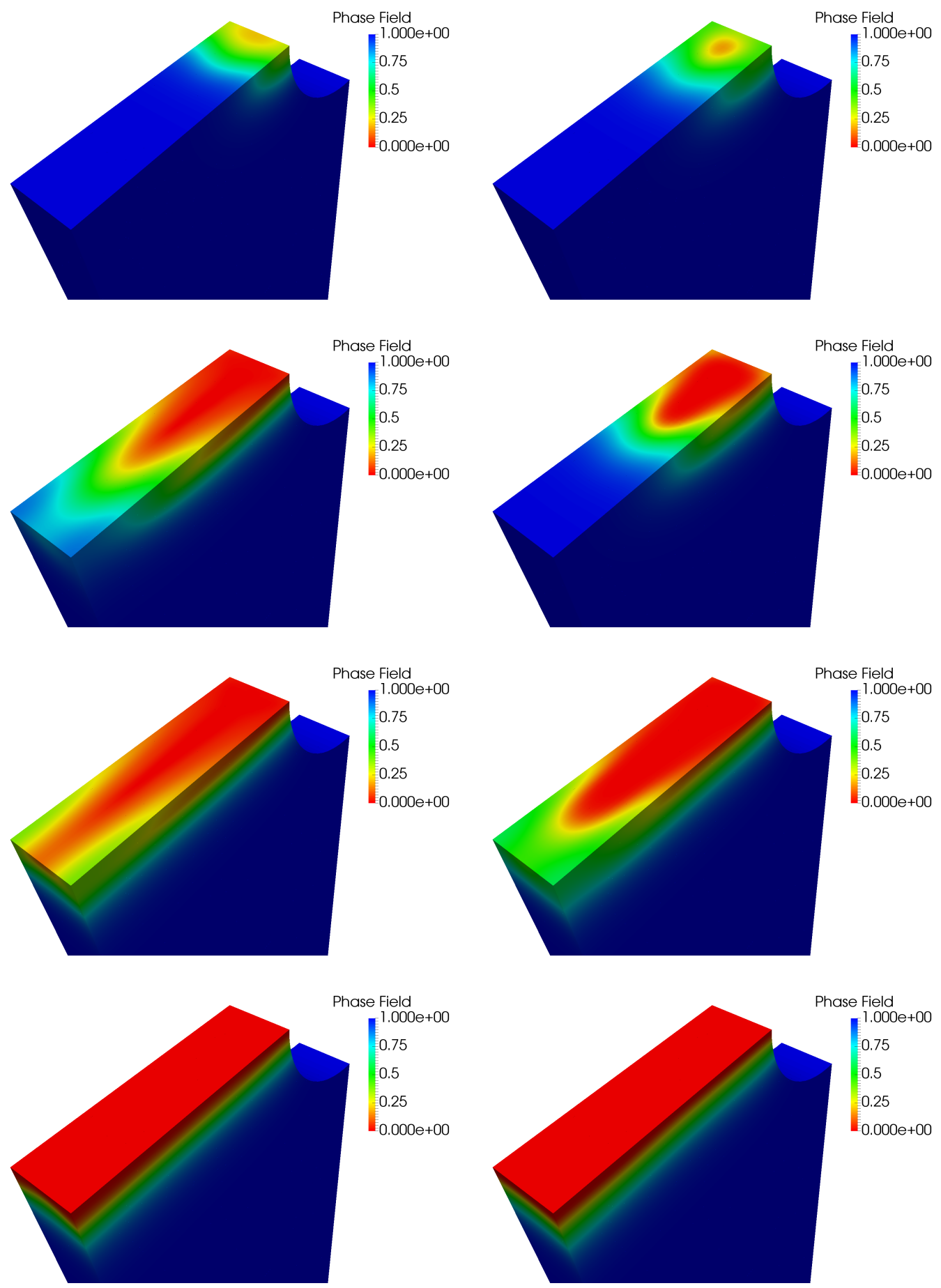

No triaxiality

With triaxiality

Figure 13: Contour plots of the phase-field as the crack progresses through a horizontal plane through the minimum cross-section area using the cubic degradation function. The left column shows the results when triaxiality is not considered and the right column shows the results when triaxiality is included. 


\subsection{Bolted Plate}

In this example we present numerical results of simulations of a circular steel plate subjected to a blast load. These simulations are based on an underwater explosion experiment performed on a steel plate that was bolted to a reaction frame. A simplified model has been used for the analysis as shown in Figure 14. In this model, the reaction frame has been replaced by a penalty term. Details of the bolted connections between the plate and reaction frame, however, have been included in the analysis model. A detailed cross-section view of the bolted connection is shown in Figure 15. The discretization is continuous between the bolt head and washer and between the washer and steel plate. Furthermore, the barrel of the bolt passes freely through the hole in the washer and plate and contact here is ignored, i.e., the barrel of the bolt is allowed to penetrate the washer and plate. These simplifications remove the need to include contact and reduces the computation complexity. Fixed displacement boundary conditions are applied to the end of the bolt.

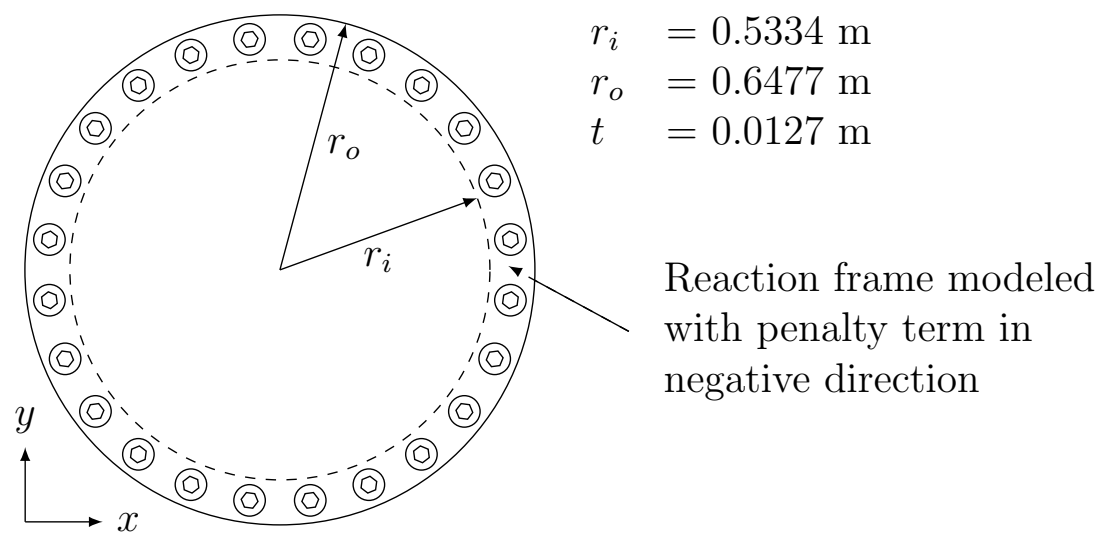

Figure 14: Plan view of the simplified analysis model with dimensions of the steel plate. The reaction frame is modeled by penalizing displacements in the negative-z direction on the back side of the plate in the outer annulus between $r_{i}$ and $r_{o}$.

The geometry of the analysis model is constructed as a quadratic multi-patch NURBS as shown in Figure 16. This allows the geometry to be represented exactly in the analysis model. A detailed view of the Bézier representation of the multi-patch NURBS description of a bolt is shown in Figure 16. We note that the number of degrees-of-freedom for this problem can be greatly reduced by the use of hierarchical refinement schemes as described by Schillinger et al. [40] and Scott et al. [41], but this has not been considered in our calculations.

The material properties used in the analysis model are given in Table 4, and the quadratic degradation function is used in all computations for this problem. The blast load is approximated as a radially distributed pressure impulse that is applied to the front surface of the plate in the 


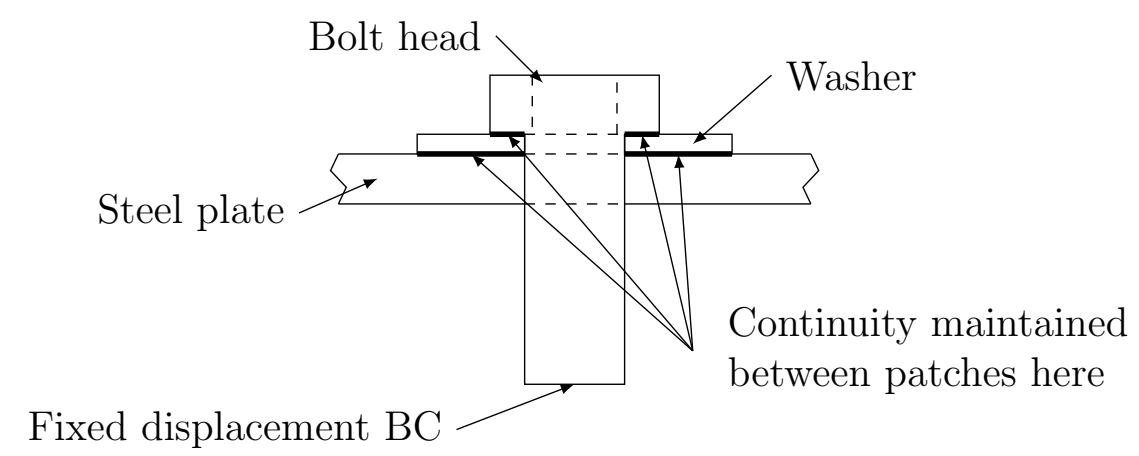

Figure 15: Detailed cross-section of a bolt connection. To simplify the computation, the bolt head is connected to the washer and the washer is connected to the steel plate in the discretization. The barrel of the bolt passes freely through the hole in the washer and plate and contact between the bolt and the washer or plate is not considered during the simulation. A fixed displacement boundary condition is applied to the end of the bolt. The length of the barrel of the bolt in the analysis model is 2.5 inches. The other dimensions of the bolt are based on a standard 1 inch diameter bolt.

current configuration. The non-dimensional radial distribution of the pressure, $g$, is defined as a function of the distance $r$ (in meters) measured from the center of the plate as

$$
g(r)=\exp \left(\frac{-r^{2}}{2 \times 0.3^{2}}\right) .
$$

The temporal distribution of the impulsive blast load is

$$
\begin{aligned}
& \tilde{p}_{0}(t)=t \cdot \exp \left(1-t \cdot 50 \times 10^{3} / s\right) \cdot 2.25 \times 10^{6} \mathrm{MPa} / \mathrm{s} \\
& p_{0}(t)=\tilde{p}_{0}(t)+ \begin{cases}0 & 0 \leq t<20 \mu \mathrm{s} \\
\left(1.0+\sin \left(\frac{\pi\left(t-2 \times 10^{-5} s\right)}{10^{-4} s-2 \times 10^{-5} s}-\frac{\pi}{2}\right)\right) \cdot 1 \mathrm{MPa} & 20 \mu \mathrm{s} \leq t<100 \mu \mathrm{s} . \\
2 \mathrm{MPa} & t \geq 100 \mu \mathrm{s}\end{cases}
\end{aligned}
$$

Plots of these functions are shown in Figure 17. The pressure is given by

$$
p(t, r)=p_{0}(t) * g(r)
$$

and results in a peak of $45 \mathrm{MPa}$ at the center of the plate at $t=20 \mu \mathrm{s}$.

The results for a number of simulations are shown in Figure 18. This figure compares the crack progression at three time intervals using both of the effective plastic work equations for a number of different plastic work threshold values. The $W_{0}=0$ case provides a good comparison that shows how introducing the triaxiality modification to the effective plastic work term effects the crack 


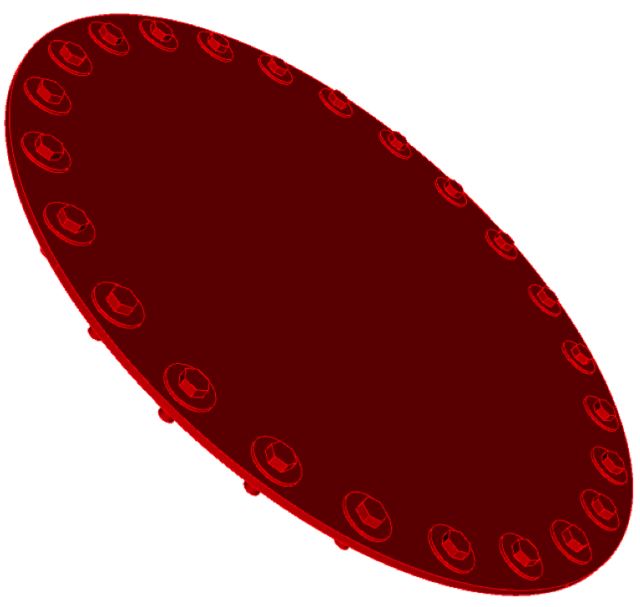

(a)

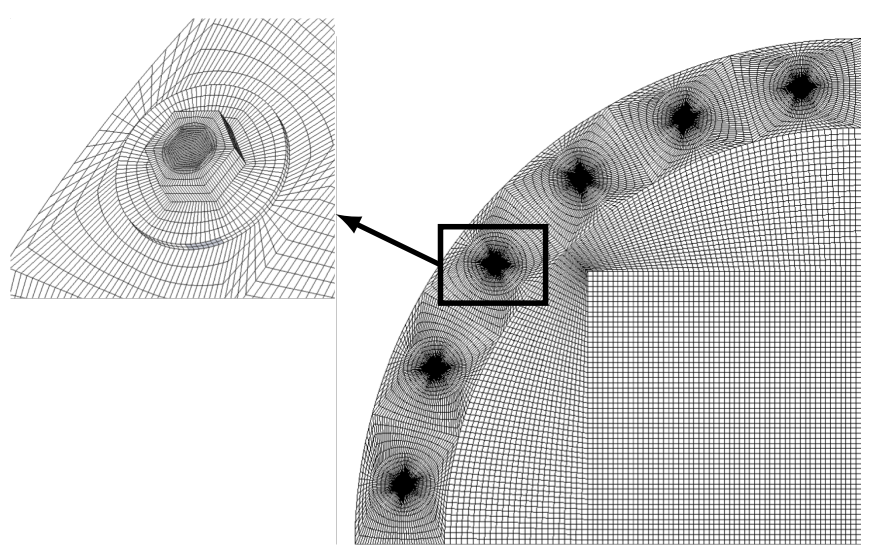

(b)

Figure 16: (a) shows an oblique angle of the geometry used for the analysis. (b) shows details of the Bézier representation of the multi-patch quadratic NURBS that describes the geometry with a close-up view of one of the bolt heads. $C^{0}$ continuity is maintained between the patches.

Table 4: Material properties.

\begin{tabular}{rl}
\hline Young's modulus & $206 \mathrm{GPa}$ \\
Poisson's ratio & 0.281 \\
Mass density & $7780 \mathrm{~kg} / \mathrm{m}^{3}$ \\
Yield stress & $355 \mathrm{MPa}$ \\
Critical fracture energy & $10^{6} \mathrm{~J} / \mathrm{m}^{2}$ \\
$\ell_{0}$ & $1.27 \times 10^{-2} \mathrm{~m}$ \\
\hline
\end{tabular}

behavior. For the "No triaxiality" case a secondary crack begins to develop at the edge of the plate and propagates back towards the main crack as it approaches the edge. For the "With triaxiality" case this secondary crack does not develop and instead the crack branches when it reaches the area of the plate supported by the reaction frame. The "With triaxiality" case also has more damage around the perimeter of the plate. For the $W_{0}=20 \mathrm{MPa}$ case the crack begins to propagate sooner for the "With triaxiality" case, which agrees with the behavior seen in the previous sections. Setting $W_{0}=40 \mathrm{MPa}$ for the "With triaxiality" case results in a crack progression that more closely matches the "No triaxiality" case with $W_{0}=20 \mathrm{MPa}$.

A detailed view of the deformed configuration of the "No triaxiality" case with $W_{0}=20 \mathrm{MPa}$ is shown in Figure 19. This figure shows that the phase-field model we are using is able to capture localized ductile behavior, which qualitatively compares well with experimental results (see for 

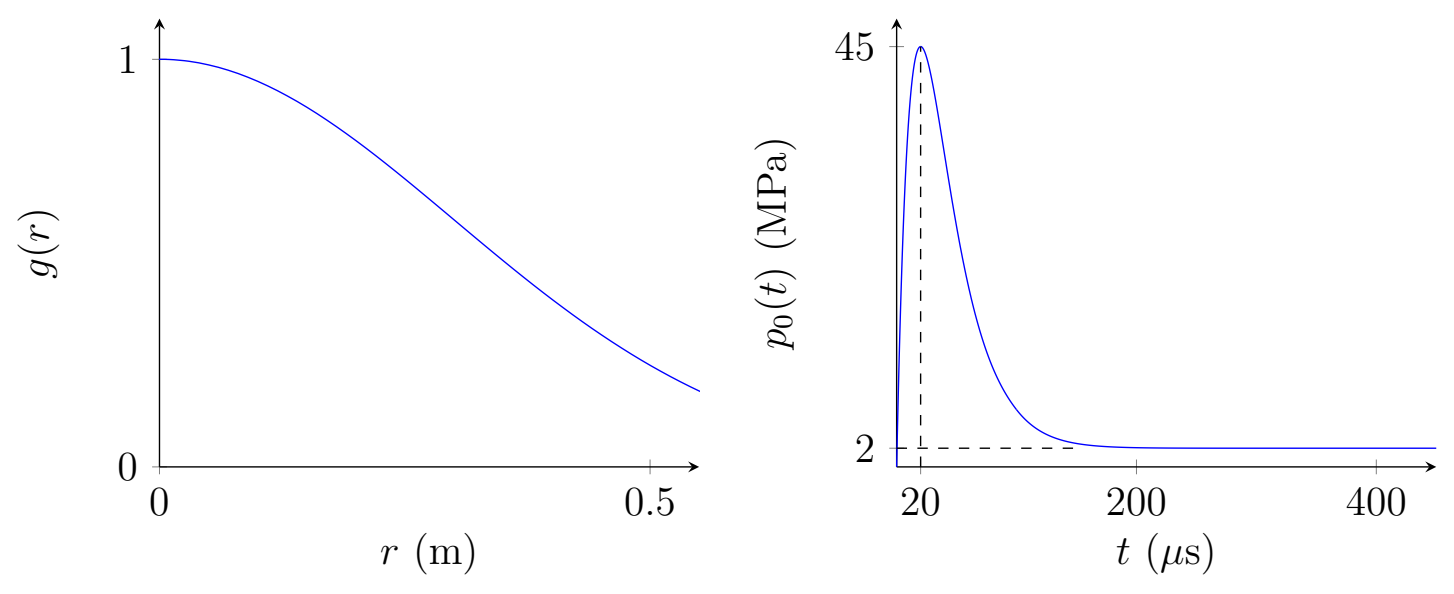

Figure 17: Plots of the radial (left) and temporal (right) functions that define the pressure impulse.

example Webster [46]).

Figure 20 shows that in the computational results the bolts immediately adjacent to the cracks fractured in tension. In Figure 21, depicting the experiment, it may be noted that several of the bolts immediately adjacent to the cracks are still present in the plate after its separation from the test frame. Our interpretation is that these bolts also fractured in tension and did not need to be pulled out in order to separate the plate from the test frame. In the experiment, all the other bolts are gone, indicating they had to be removed in order to separate the plate from the test frame. 


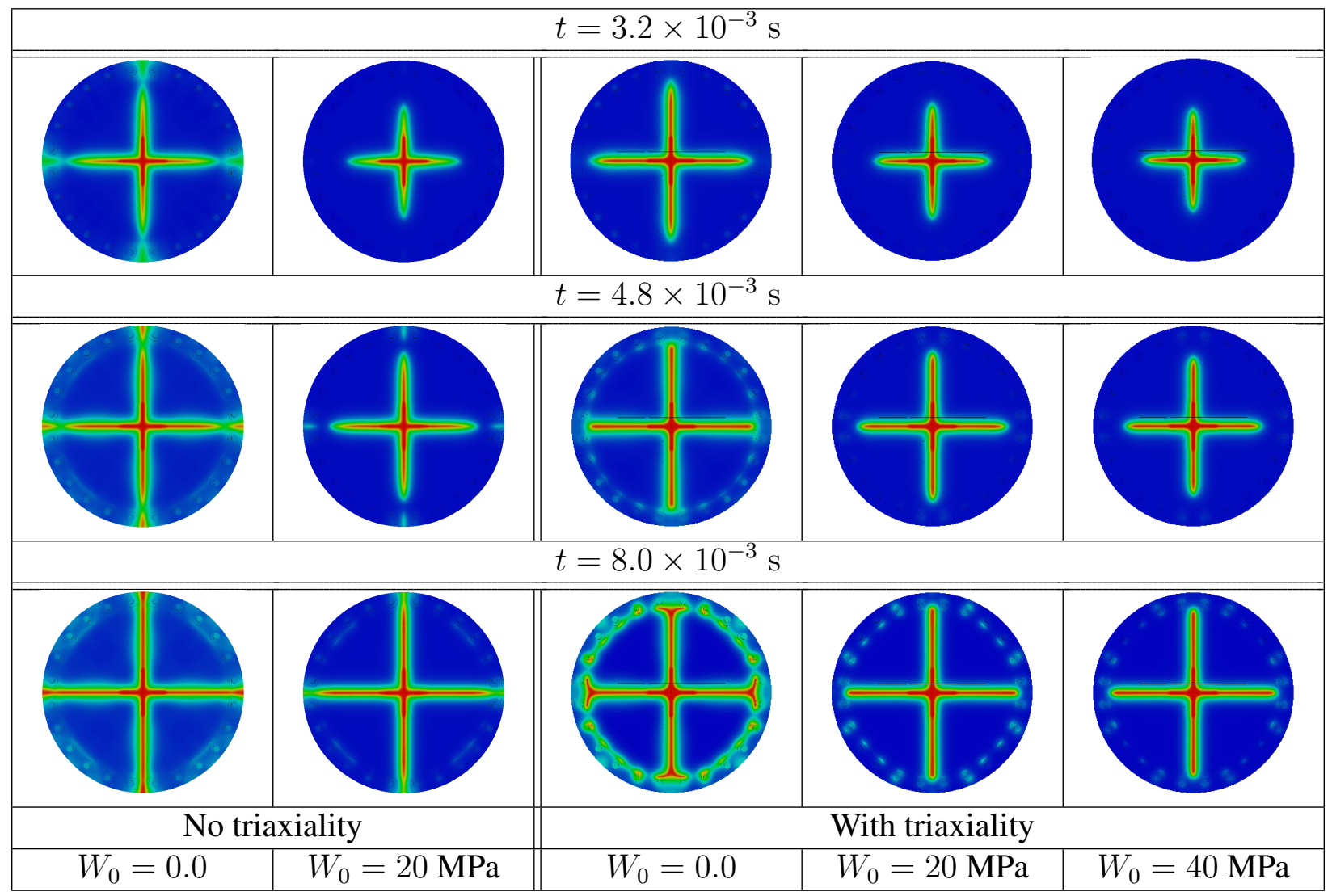

Figure 18: A comparison of crack progression at three time steps for different values of $W_{0}$ with and without the triaxiality modification to the effective plastic work term.

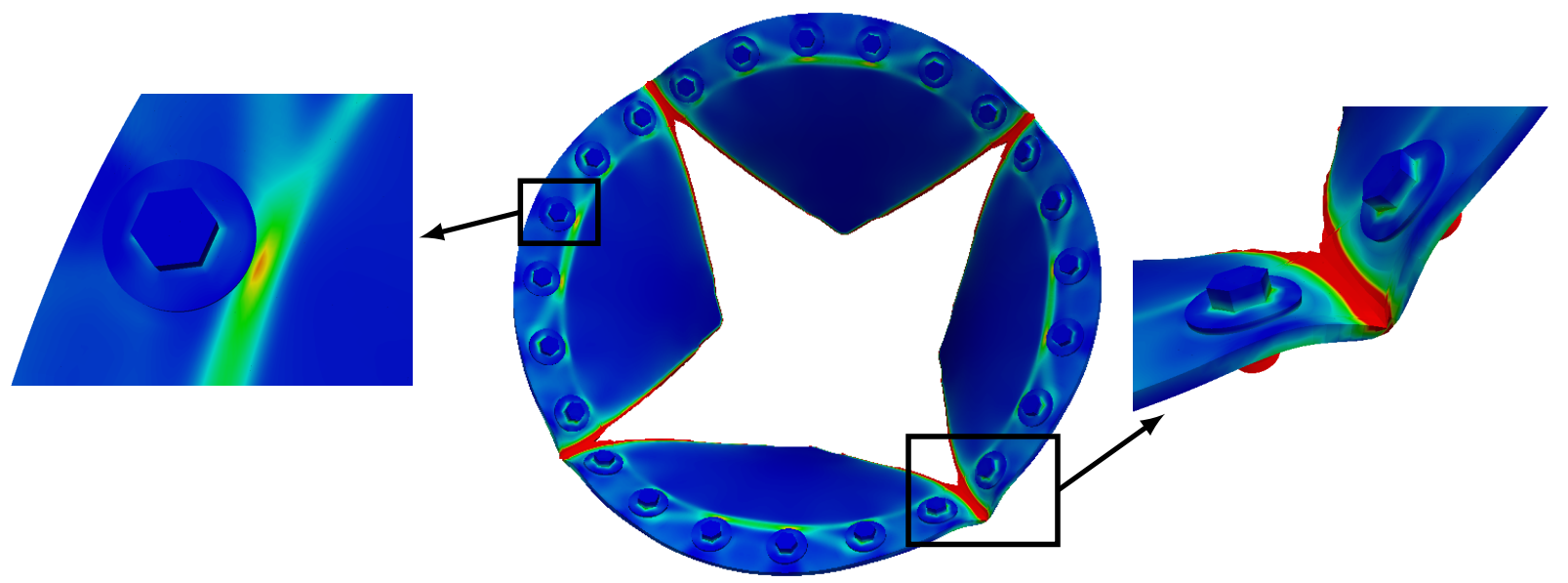

Figure 19: Details of the deformed plate for computations with $W_{0}=20 \mathrm{MPa}$. The color plots indicate the level of plastic yielding. On the left, we see the effect of a bolt hole on the distribution of plastic yielding. On the right we see that the plate begins to fold as the crack nears the edge. This behavior matches well qualitatively with experimental results. 


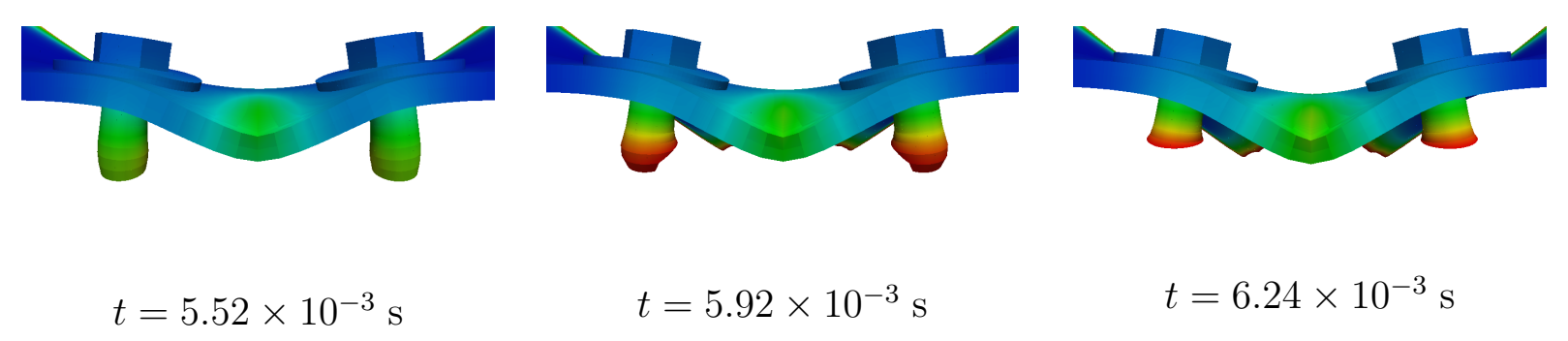

Figure 20: A detailed view showing the progression of the failure of the bolts for the computational with $W_{0}=20 \mathrm{MPa}$. The color scale indicates the value of the phase-field.
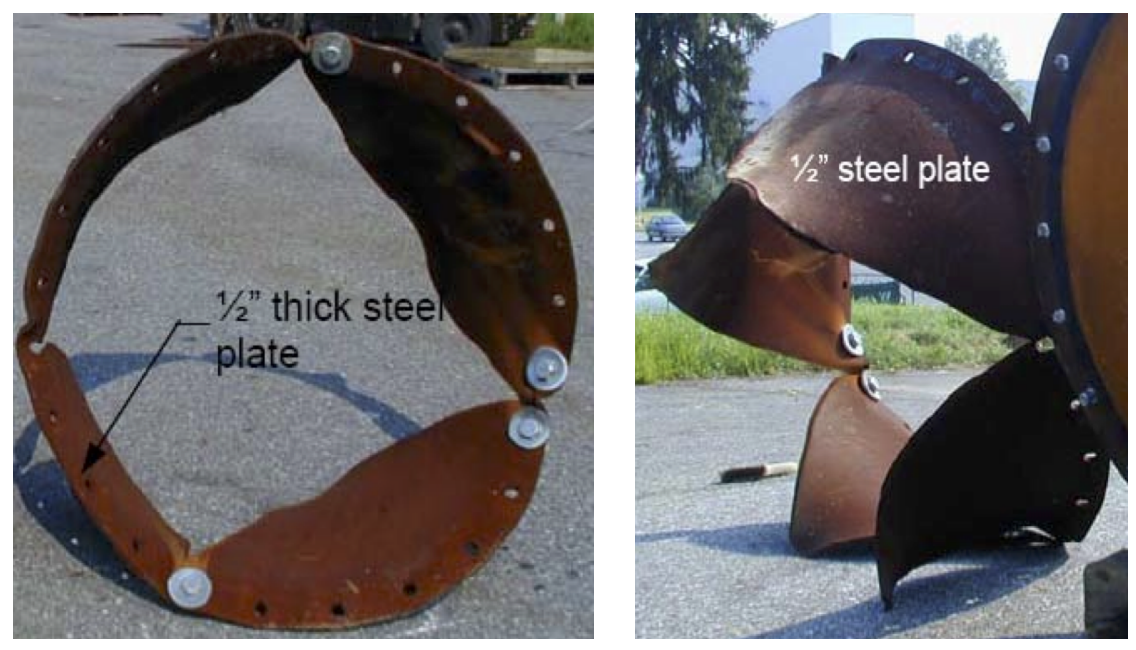

Figure 21: Figures showing the experimental results from Webster [46]. 


\subsubsection{A note on boundary conditions}

Building detailed representations of boundary conditions and other geometric details in the analysis model is often time consuming and may increase computational cost. Because of this, simplifications are often made where details that are deemed unimportant are removed from the analysis model. Care must be taken, however, as too much simplification will lead to poor results. For example, Figure 22 shows a simplified representation of the plate presented in the previous section where the bolts have been removed. For this geometry the reaction frame is modeled by applying displacement boundary conditions to the annular surfaces between $r_{i}$ and $r_{0}$. We have computed results on this simplified model using two different representations of the reaction frame as shown in Figure 23. Clamped and sliding boundary conditions, where all displacements where constrained or the surface was free to move in the $x-y$ plane respectively, were applied. Comparing the results with those computed with the bolts represented in the analysis model it is clear that the detailed representation including the bolts is critical in order to achieve accurate results.

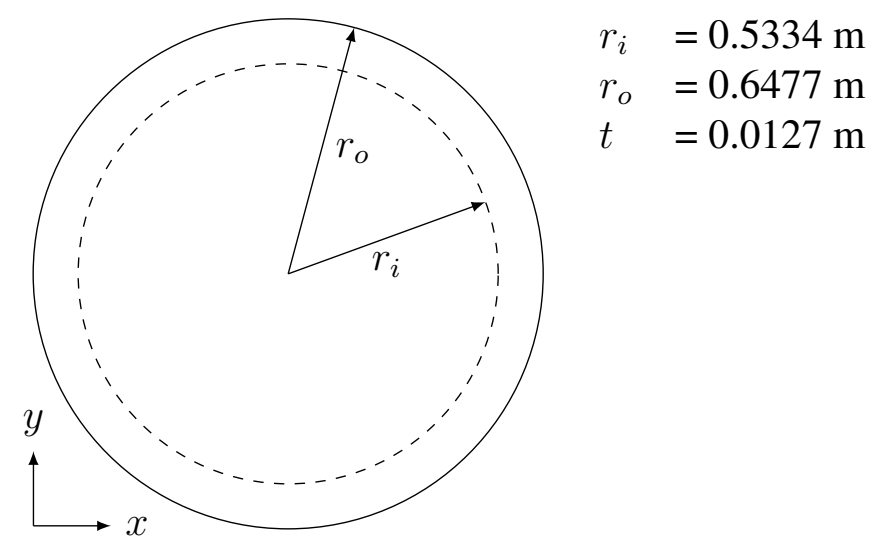

Figure 22: A simplified model where the bolt details have been removed. The reaction frame was modeled by setting Dirichlet boundary conditions on both surfaces of the plate between $r_{i}$ and $r_{o}$. A clamped boundary condition was applied by fixing all displacements on these surfaces. A sliding boundary conditions was applied by fixing the out of plane displacements on these surfaces.

\section{Conclusion}

In this work we have presented several innovations to the phase-field modeling of fracture that improve upon its representation of fracture in ductile material behavior. Our theoretical framework is based on a microforce balance and a general energy potential accounting for large deformation elastic-plastic response and plastic softening. We have developed a cubic degradation function 


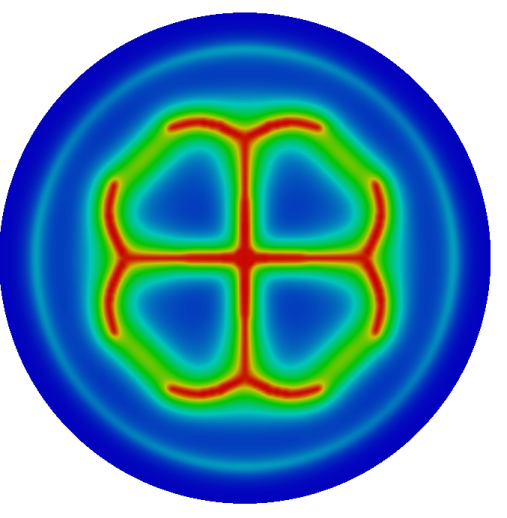

Clamped

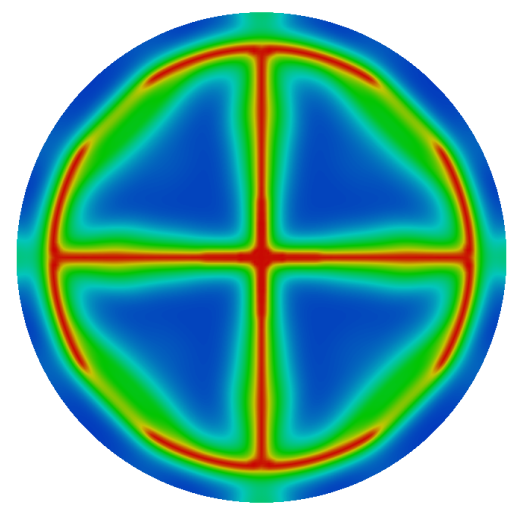

Sliding

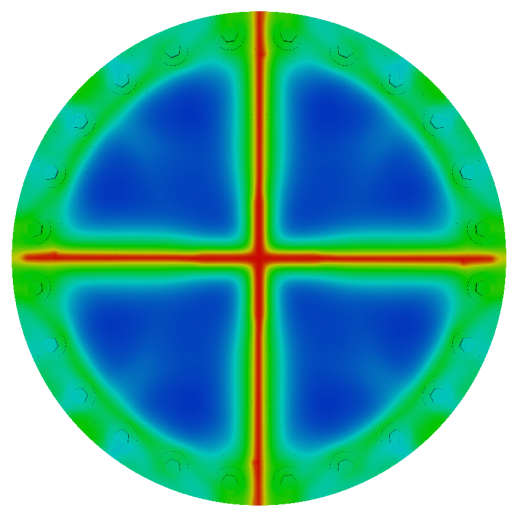

Bolted

Figure 23: Results using a different representations of the connection with the reaction frame. The clamped condition constrains all displacement in the annular surface between $r_{i}$ and $r_{o}$. For the sliding boundary condition the annular surface is free to move in the $x-y$ plane. The bolted connection is described in the previous section. Only the bolted configuration gives results consistent with experiments.

that provides a nearly linear stress-strain response prior to crack initiation in elastic bodies, and considerably improves upon shortcomings noted for the widely used quadratic degradation function. The cubic degradation function also attains a given critical stress with a larger length scale than for the quadratic function, permitting coarser meshes to be used and greater efficiency. We have introduced a plastic yield surface degradation function that accounts for plastic softening as cracks develop, eliminates non-physical elastic deformations after crack initiation, and leads to better representation of the physical processes that occur during failure. We have also provided a mechanism that allows for stress triaxiality to influence crack initiation and growth. Finally, we have also identified and corrected a shortcoming in a widely used return-mapping implementation of a hyperelastic-plastic large deformation constitutive model that can lead to non-physical behavior. Several examples have been presented identifying the beneficial effects that these innovations have on computed results.

Although much work remains to be done to fully validate the use of phase-field models for predicting ductile fracture, the results presented here are encouraging and indicate that this approach, at the very least, provides qualitative agreement with expected physical behavior in complex physical situations and has demonstrated considerable potential for practical engineering computations. 


\section{Acknowledgements}

This work was partially supported by Grants from the Office of Naval Research under contract number N00014-08-1-0992 and the Army Research Office under contract number W911NF-101-0216. T.J.R. Hughes, A. Anvari, and C.M. Landis were also supported by ARO grant number W911NF-13-1-0220. This support is gratefully acknowledged.

The authors also acknowledge the Texas Advanced Computing Center (TACC) at The University of Texas at Austin for pro- viding HPC and visualization resources that have contributed to the research results reported within this paper. URL: http:// www.tacc.utexas.edu.

\section{A Elastoplastic constitutive model}

Here we present the details of the associative elastoplastic constitutive model based on $J_{2}$ flow theory with isotropic hardening that was introduced in Section 3.2. We assume an isotropic stress response governed by the damage/elastic constitutive model summarized in Section 3.4.2. We also assume the plastic flow to be isochoric, i.e.,

$$
\operatorname{det} \mathbf{F}^{p}=1 \Rightarrow J=\operatorname{det} \mathbf{F}^{e}
$$

The associative flow rule is derived by making use of the principle of maximum plastic dissipation. This principle states that for any given fixed internal plastic variables $\left\{\mathbf{C}^{p}, \mathbf{Q}\right\}$ and yield function $f\left(\mathbf{C}, \mathbf{C}^{p}, \mathbf{Q}\right)$ the actual deformation tensor, $\mathbf{C}$, satisfies

$$
\begin{aligned}
& \mathbb{D}^{p}\left(\mathbf{C}, \mathbf{C}^{p}, \mathbf{Q} ; \dot{\mathbf{C}}^{p}, \dot{\mathbf{Q}}\right) \geq \mathbb{D}^{p}\left(\hat{\mathbf{C}}, \mathbf{C}^{p}, \mathbf{Q} ; \dot{\mathbf{C}}^{p}, \dot{\mathbf{Q}}\right) \\
& \quad \text { for all } \hat{\mathbf{C}} \text { s.t. } \quad f\left(\hat{\mathbf{C}}, \mathbf{C}^{p}, \mathbf{Q}\right) \leq 0
\end{aligned}
$$

where $\mathbb{D}^{p}$ is defined in (34). Assuming an uncoupled free energy function in the internal variables, $\mathrm{Q}$, the principle of maximum plastic dissipation leads to the associative flow rule

$$
-4 \frac{\partial^{2} W}{\partial \mathbf{C} \partial \mathbf{C}^{p}}: \dot{\mathbf{C}}^{p}=2 \dot{\gamma} \frac{\partial f}{\partial \mathbf{C}}
$$

and the Karush-Kuhn-Tucker (KKT) loading/unloading conditions

$$
\dot{\gamma} \geq 0, \quad f \leq, \quad \dot{\gamma} f=0
$$

For a detailed derivation see Simo [42]. 
For the current formulation, we assume that yielding is governed by the Mises-Huber yield condition, given in terms of the Kirchhoff stress $\tau$. The yield function for this case is written as

$$
f(\boldsymbol{\tau}, \alpha)=\|\mathbf{s}\|-\sqrt{\frac{2}{3}} k(\alpha)
$$

where $\alpha$ is the hardening parameter and $k(\alpha)$ the isotropic hardening modulus. The hardening law is determined by the choice of $k(\alpha)$. For example,

$$
k(\alpha)= \begin{cases}\sigma_{y} & (\text { Perfect plasticity }) \\ \sigma_{y}+K \alpha & (\text { Linear hardening })\end{cases}
$$

where $\sigma_{y}$ is the flow stress and $K$ is the linear hardening coefficient.

Given the hyperelastic stress response summarized in Section 3.4.2 the yield function can be rewritten in the form $f\left(\overline{\mathbf{C}}, \mathbf{C}^{p}, \mathbf{Q}\right)$. Then, again following the derivation given by Simo [42], the hardening law is

$$
\dot{\alpha}=\sqrt{\frac{2}{3}} \dot{\gamma}
$$

and the flow rule is

$$
J^{-2 / 3} \operatorname{DEV}\left[2 \frac{\partial^{2} g(c) \bar{W}}{\partial \overline{\mathbf{C}} \partial \mathbf{C}^{p}}: \dot{\mathbf{C}}^{p}\right]=-\dot{\gamma} J^{-2 / 3} \operatorname{DEV}\left[\frac{\partial f}{\partial \overline{\mathbf{C}}}\right]
$$

where $\operatorname{DEV}[\cdot]=(\cdot)-\frac{1}{3}[\mathbf{C}:(\cdot)] \mathbf{C}^{-1}$ is the deviator in the reference configuration. Expanding the differential term on the right-hand side of this equation we get

$$
\frac{\partial f}{\partial \overline{\mathbf{C}}}=\frac{\partial}{\partial \overline{\mathbf{C}}}\left(g(c) \mu \operatorname{dev}\left[\overline{\mathbf{b}}^{e}\right]\right)
$$

Since we use a staggered solution scheme we assume that $c$ is fixed during plastic flow allowing $g(c)$ to be factored out of both sides of (99). This leads to a flow rule that is independent of $c$. In the reference configuration, the flow rule simplifies to

$$
\begin{aligned}
\operatorname{DEV}\left[\dot{\mathbf{C}}^{p-1}\right] & =-\frac{2}{3} \dot{\gamma} \mathbf{C}: \mathbf{C}^{p-1} \mathbf{N} \\
\mathbf{N} & =\mathbf{F}^{-1} \mathbf{n} \mathbf{F}^{-T} \\
\mathbf{n} & =\frac{\mathbf{s}}{\|\mathbf{s}\|}
\end{aligned}
$$


which can be pushed forward to the current configuration to get

$$
\operatorname{dev}\left[L_{v} \mathbf{b}^{e}\right]=-\frac{2}{3} \dot{\gamma} \operatorname{tr}\left[\mathbf{b}^{e}\right] \mathbf{n}
$$

where $L_{v} \mathbf{b}^{e}$ is the Lie derivative of $\mathbf{b}^{e}$ (see Simo and Hughes [43] for details). We point out that the principle of maximum plastic dissipation determines only the deviatoric part of $\dot{\mathbf{C}}^{p-1}$ and $L_{v} \mathbf{b}^{e}$. The spherical part of these tensors must be determined based on the isochoric assumption, i.e. $J^{e}=\operatorname{det}[\mathbf{F}]$ and $\operatorname{det}\left[\overline{\mathbf{b}}^{e}\right]=1$. The complete elastic/plastic constitutive model is summarized in Section 3.4.3.

\section{B Return-mapping algorithm}

Numerical integration of the constitutive theory summarized in Section 3.4.3 is performed by a backward Euler scheme that leads to the construction of a return-mapping algorithm. Following the procedure outlined by Simo and Hughes [43, Chapter 9] and taking into account the definition of the damage/elastic stress response from Section 3.4.2, the phase-field parameter is incorporated as follows. For each time interval, $\left[t_{n}, t_{n+1}\right]$, we assume the initial states $\phi_{n}, \overline{\mathbf{b}}_{n}^{e}$, $\alpha_{n}$, and $\mathbf{F}_{n}$ are known. The configuration at time $t_{n+1}$ is obtained from the incremental displacement field $\mathbf{u}_{n}: \Omega_{n} \rightarrow \mathbb{R}^{3}$ as

$$
\phi_{n+1}(\mathbf{X})=\phi_{n}(\mathbf{X})+\mathbf{u}_{n}\left(\phi_{n}(\mathbf{X})\right)
$$

and the relative deformation gradient is given by

$$
\boldsymbol{f}_{n+1}=\mathbf{I}+\nabla_{x_{n}} \mathbf{u}_{n}
$$

Assuming an elastic trial response, i.e., $\alpha_{n+1}^{\text {trial }}=\alpha_{n}$, the trial deformation is given by

$$
\begin{aligned}
& \overline{\boldsymbol{f}}_{n+1}=\operatorname{det}\left[\boldsymbol{f}_{n+1}\right]^{-1 / 3} \boldsymbol{f}_{n+1} \\
& \overline{\mathbf{b}}_{n+1}^{e \text { trial }}=\overline{\boldsymbol{f}}_{n+1} \overline{\mathbf{b}}_{n}^{e} \overline{\boldsymbol{f}}_{n+1}^{T} .
\end{aligned}
$$

The trial damaged deviatoric stress is then computed from the relationship for the damaged Kirchhoff stress, $\tau=J \sigma$, as

$$
\mathbf{s}_{n+1}^{\text {trial }}=\operatorname{dev}\left[\boldsymbol{\tau}_{n+1}^{\text {trial }}\right]=g(c) \mu \operatorname{dev}\left[\overline{\mathbf{b}}_{n+1}^{e \text { trial }}\right]
$$


Now, letting $\Delta \gamma$ be the algorithmic counterpart to $\dot{\gamma}$, the discrete KKT loading/unloading conditions are

$$
f\left(\boldsymbol{\tau}_{n+1}, \alpha_{n+1}\right) \leq 0, \quad \Delta \gamma \geq 0, \quad \Delta \gamma f\left(\boldsymbol{\tau}_{n+1}, \alpha_{n+1}\right)=0
$$

These conditions give rise to two alternatives based on the result of the evaluation of the yield function for the given trial state. We define

$$
f_{n+1}^{\text {trial }}:=f\left(\boldsymbol{\tau}_{n+1}^{\text {trial }}, \alpha_{n+1}^{\text {trial }}\right)=\left\|\mathbf{s}_{n+1}^{\text {trial }}\right\|-\sqrt{\frac{2}{3}} k\left(\alpha_{n+1}^{\text {trial }}\right)
$$

If $f_{n+1}^{\text {trial }} \leq 0$, then the KKT conditions are satisfied with $\Delta \gamma=0$ and the trial damage/elastic state is accepted as the solution at $t_{n+1}$. If, on the other hand, $f_{n+1}^{\text {trial }} \geq 0$, then the KKT conditions are not satisfied, implying that the trial stress has moved outside the yield surface and is not admissible. In this case, we need to update $\alpha_{n+1}$ and $\tau_{n+1}$ to return the stress to the yield surface. These updates are determined by a backward Euler discetization of the evolution equations, which leads to

$$
\begin{aligned}
\operatorname{dev}\left[\overline{\mathbf{b}}_{n+1}^{e}\right] & =\operatorname{dev}\left[\overline{\mathbf{b}}_{n+1}^{e \text { trial }}\right]-\frac{2}{3} \Delta \gamma \operatorname{tr}\left[\overline{\mathbf{b}}_{n+1}^{e}\right] \mathbf{n}_{n+1} \\
\alpha_{n+1} & =\alpha_{n}+\sqrt{\frac{2}{3}} \Delta \gamma
\end{aligned}
$$

where

$$
\mathbf{n}_{n+1}=\frac{\mathbf{s}_{n+1}}{\left\|\mathbf{s}_{n+1}\right\|}
$$

Using these update equations the new admissible state is determined by finding $\Delta \gamma>0$ and $\operatorname{tr}\left[\overline{\mathbf{b}}_{n+1}^{e}\right]$ such that $f\left(\boldsymbol{\tau}_{n+1}, \alpha_{n+1}\right)=0$ and $\operatorname{det}\left[\overline{\mathbf{b}}_{n+1}^{e}\right]=1$.

To derive the system of equations that will be solved to find the admissible state we first find a relationship between $\left\|\mathbf{s}_{n+1}\right\|$ and $\left\|\mathbf{s}_{n+1}^{\text {trial }}\right\|$. This is done by substituting (112) into the constitutive relationship (109) to get

$$
\mathbf{s}_{n+1}=\mathbf{s}_{n+1}^{\text {trial }}-\frac{2}{3} g(c) \mu \Delta \gamma \operatorname{tr}\left[\overline{\mathbf{b}}_{n+1}^{e}\right] \mathbf{n}_{n+1}
$$

which can be rearranged to get

$$
\mathbf{s}_{n+1}+\frac{2}{3} g(c) \mu \Delta \gamma \operatorname{tr}\left[\overline{\mathbf{b}}_{n+1}^{e}\right] \mathbf{n}_{n+1}=\mathbf{s}_{n+1}^{\text {trial }}
$$


Now recongnizing that $\mathbf{s}_{n+1}=\left\|\mathbf{s}_{n+1}\right\| \mathbf{n}_{n+1}$ we can rewrite this equation one more time as

$$
\left(\left\|\mathbf{s}_{n+1}\right\|+\frac{2}{3} g(c) \mu \Delta \gamma \operatorname{tr}\left[\overline{\mathbf{b}}_{n+1}^{e}\right]\right) \mathbf{n}_{n+1}=\left\|\mathbf{s}_{n+1}^{\text {trial }}\right\| \mathbf{n}_{n+1}^{\text {trial }}
$$

We also have from (116) that

$$
\begin{aligned}
\left\|\mathbf{s}_{n+1}+\frac{2}{3} g(c) \mu \Delta \gamma \operatorname{tr}\left[\overline{\mathbf{b}}_{n+1}^{e}\right] \mathbf{n}_{n+1}\right\| & =\left\|\mathbf{s}_{n+1}\left(1+\frac{2}{3} g(c) \mu \Delta \gamma \operatorname{tr}\left[\overline{\mathbf{b}}_{n+1}^{e}\right] \frac{1}{\left\|\mathbf{s}_{n+1}\right\|}\right)\right\| \\
& =\left\|\mathbf{s}_{n+1}\right\|+\frac{2}{3} g(c) \mu \Delta \gamma \operatorname{tr}\left[\overline{\mathbf{b}}_{n+1}^{e}\right] \\
& =\left\|\mathbf{s}_{n+1}^{\text {trial }}\right\| .
\end{aligned}
$$

This implies that $\mathbf{n}_{n+1},=\mathbf{n}_{n+1}^{\text {trial }}$ and we arrive at the relationship we were seeking:

$$
\left\|\mathbf{s}_{n+1}\right\|=\left\|\mathbf{s}_{n+1}^{\text {trial }}\right\|-\frac{2}{3} g(c) \mu \Delta \gamma \operatorname{tr}\left[\overline{\mathbf{b}}_{n+1}^{e}\right]
$$

Substituting this equation and (113) into the yield function we can then find $\Delta \gamma$ and $\operatorname{tr}\left[\overline{\mathbf{b}}_{n+1}^{e}\right]$ by solving the system of scalar valued equations

$$
\begin{aligned}
& \hat{f}(\Delta \gamma)=\left\|\mathbf{s}_{n+1}^{\text {trial }}\right\|-\frac{2}{3} g(c) \mu \Delta \gamma \operatorname{tr}\left[\overline{\mathbf{b}}_{n+1}^{e}\right]-\sqrt{\frac{2}{3}} k\left(\alpha_{n}+\sqrt{\frac{2}{3}} \Delta \gamma\right)=0 \\
& \operatorname{det}\left[\overline{\mathbf{b}}_{n+1}^{e}\right]=\operatorname{det}\left[\operatorname{dev}\left[\overline{\mathbf{b}}_{n+1}^{\text {etrial }}\right]+\frac{1}{3} \operatorname{tr}\left[\overline{\mathbf{b}}_{n+1}^{e}\right]\left(\mathbf{I}-2 \Delta \gamma \mathbf{n}_{n+1}^{\text {trial }}\right)\right]=1 .
\end{aligned}
$$

We note that our derivation has resulted in a slightly different update formula than what has been presented in Simo [42] and Simo and Hughes [43]. The difference stems from an assumption in these works that leads to the condition $\operatorname{tr}\left[\overline{\mathbf{b}}_{n+1}^{e}\right]=\operatorname{tr}\left[\overline{\mathbf{b}}_{n+1}^{e \text { trial }}\right]$ (in [43] this is a result of dropping the deviatoric operators from (112) to get $\overline{\mathbf{b}}_{n+1}^{e}=\overline{\mathbf{b}}_{n+1}^{e \text { trial }}-\frac{2}{3} \Delta \gamma \operatorname{tr}\left[\overline{\mathbf{b}}_{n+1}^{e}\right] \mathbf{n}_{n+1}$ and then taking the trace of both sides of this equation). This leads to an update that depends on the solution of the single scalar valued function

$$
\left\|\mathbf{s}_{n+1}^{\text {trial }}\right\|-\frac{2}{3} g(c) \mu \Delta \gamma \operatorname{tr}\left[\overline{\mathbf{b}}_{n+1}^{e \text { trial }}\right]-\sqrt{\frac{2}{3}} k\left(\alpha_{n}+\sqrt{\frac{2}{3}} \Delta \gamma\right)=0
$$

(note that the left-hand side depends only on the trial state of $\overline{\mathbf{b}}^{e}$ ). This difference is subtle and has almost no effect (numerically) on the deviatoric part of $\overline{\mathbf{b}}^{e}$ but leads to an accumulation of error in its trace. We illustrate this by a simple exercise of the algorithm presented in Box 9.1 of Simo and Hughes [43, Chapter 9], which is summarized in Box 1. We choose $\mu=25 \times 10^{9}, \kappa=75 \times 10^{9}$, and 
$k\left(\alpha_{n}\right)=\sigma_{y}=448 \times 10^{6}$ for the material parameters and start with an undeformed configuration, $\mathbf{F}_{0}=\mathbf{I}$. We then specify a relative deformation gradient

$$
\boldsymbol{f}_{1}=\left[\begin{array}{ccc}
1.01 & 0 & 0 \\
0 & 0.999 & 0 \\
0 & 0 & 0.999
\end{array}\right]
$$

which causes plastic loading in Step 3 of the algorithm. Following the return-mapping and stress update in Steps 4 and 5 we compute the determinate of $\overline{\mathbf{b}}_{1}^{e}$ and get 1.00005 . Recall from the definition of $\overline{\mathbf{b}}^{e}$ that its determinate should be equal to one.

There are two sources of this error in the algorithm. The first is the use of the trace of $\overline{\mathbf{b}}_{n+1}^{e \text { trial }}$ instead of $\overline{\mathbf{b}}_{n+1}^{e}$ in the left-hand side of (124). This error effects only the devaitoric part of $\overline{\mathbf{b}}^{e}$ and in practice has almost no effect on the numerical results. This claim is supported by nearly 30 years of use of this model where the interest has been on the stress-strain response, which during plastic flow is governed by the evolution of the deviatoric part of $\overline{\mathbf{b}}^{e}$.

The second source of error follows from the condition $\operatorname{tr}\left[\overline{\mathbf{b}}_{n+1}^{e}\right]=\operatorname{tr}\left[\overline{\mathbf{b}}_{n+1}^{e \text { trial }}\right]$. Over time, this leads to an accumulation of error in the trace of $\overline{\mathbf{b}}^{e}$ and one manifestation of this error is in the error in the determinant of $\overline{\mathbf{b}}^{e}$. If we follow the computations performed in Box 1 we see that the determinant of $\overline{\mathbf{b}}^{e}$ is never used and hence this source of error has no effect on the stress-strain response. For the phase-field model we have presented in this work, however, the evolution of the phase-field depends on the elastic strain energy, which in the current configuration is given by

$$
\bar{W}\left(\overline{\mathbf{b}}^{e}\right)=\frac{1}{2} \mu\left(\operatorname{tr}\left[\overline{\mathbf{b}}^{e}\right]-3\right) .
$$

It is obvious from this equation that errors in the trace of $\overline{\mathbf{b}}^{e}$ will lead to errors in computation of the elastic strain energy.

In light of this discussion we propose a simple modification to the algorithm presented in Box 1 for computations involving a phase-field model that depends on the evolution of the elastic strain energy. In Step 6 of this algorithm we update the trace of $\overline{\mathbf{b}}_{n+1}^{e}$ using (123) such that $\operatorname{det}\left[\overline{\mathbf{b}}_{n+1}^{e}\right]=1$. By leaving the preceding steps unmodified we make use of (124) instead of (122) to update the deviatoric part of $\overline{\mathbf{b}}^{e}$, effectively uncoupling the system composed of (122) and (123). This approximation is justified based on the preceding discussion with regards to the errors in practical computations. The modified return-mapping algorithm is summarized in Section 4.1.1. A comparison between the two return mapping algorithms for a simple uniaxial case with linear hardening is shown in Figure 24. In this figure the standard update refers to computations performed using the 
1. Update the current configuration

$$
\begin{aligned}
& \phi_{n+1}(\mathbf{X})=\phi_{n}(\mathbf{X})+\mathbf{u}_{n}\left(\phi_{n}(\mathbf{X})\right) \\
& \boldsymbol{f}_{n+1}=\mathbf{I}+\nabla_{x_{n}} \mathbf{u}_{n} \\
& \mathbf{F}_{n+1}=\boldsymbol{f}_{n+1} \mathbf{F}_{n}
\end{aligned}
$$

2. Compute the damage/elastic predictor

$$
\begin{aligned}
& \overline{\boldsymbol{f}}_{n+1}=\operatorname{det}\left[\boldsymbol{f}_{n+1}\right]^{-1 / 3} \boldsymbol{f}_{n+1} \\
& \overline{\mathbf{b}}_{n+1}^{e \text { trial }}=\overline{\boldsymbol{f}}_{n+1} \overline{\mathbf{b}}_{n}^{e} \overline{\boldsymbol{f}}_{n+1}^{T} \\
& \mathbf{s}_{n+1}^{\text {trial }}=\mu \operatorname{dev}\left[\overline{\mathbf{b}}_{n+1}^{e \text { trial }}\right]
\end{aligned}
$$

3. Check for plastic loading

$$
\begin{aligned}
& f_{n+1}^{\text {trial }}=\left\|\mathbf{s}_{n+1}^{\text {trial }}\right\|-\sqrt{\frac{2}{3}} k\left(\alpha_{n}\right) \\
& \text { IF } f_{n+1}^{\text {trial }} \leq 0 \text { THEN } \\
& \text { Set }(\cdot)_{n+1}=(\cdot)_{n+1}^{\text {trial }} \text {, and EXIT }
\end{aligned}
$$

ELSE

Go to 4 (return-mapping)

\section{ENDIF}

\section{Return-mapping}

$$
\begin{aligned}
& \bar{I}_{n+1}^{e \text { trial }}=\frac{1}{3} \operatorname{tr}\left[\overline{\mathbf{b}}_{n+1}^{e \text { trial }}\right] \\
& \bar{\mu}=\mu \bar{I}_{n+1}^{e \text { trial }}
\end{aligned}
$$

Solve: $\hat{f}(\Delta \gamma)=\left\|\mathbf{s}_{n+1}^{\text {trial }}\right\|-g_{p}\left(c_{n}\right) \sqrt{\frac{2}{3}} k\left(\alpha_{n}+\sqrt{\frac{2}{3}} \Delta \gamma\right)-2 \bar{\mu} \Delta \gamma=0$

$$
\begin{aligned}
& \mathbf{s}_{n+1}=\mathbf{s}_{n+1}^{\text {trial }}-2 \bar{\mu} \Delta \gamma \mathbf{n} \\
& \alpha_{n+1}=\alpha_{n}+\sqrt{\frac{2}{3}} \Delta \gamma
\end{aligned}
$$

5. Update the Kirchhoff stress

$$
\begin{aligned}
J_{n+1} & =\operatorname{det}\left[\mathbf{F}_{n+1}\right] \\
p_{n+1} & =U^{\prime}\left(J_{n+1}\right) \\
\boldsymbol{\tau}_{n+1} & =J_{n+1} p_{n+1} \mathbf{I}+\mathbf{s}_{n+1}
\end{aligned}
$$

6. Update the intermediate configuration

$$
\overline{\mathbf{b}}_{n+1}^{e}=\frac{\mathbf{s}_{n+1}}{\mu}+\bar{I}_{n+1}^{e \text { trial }} \mathbf{I}
$$

Box 1: Return-mapping algorithm for $J_{2}$-Flow theory from [43]. 

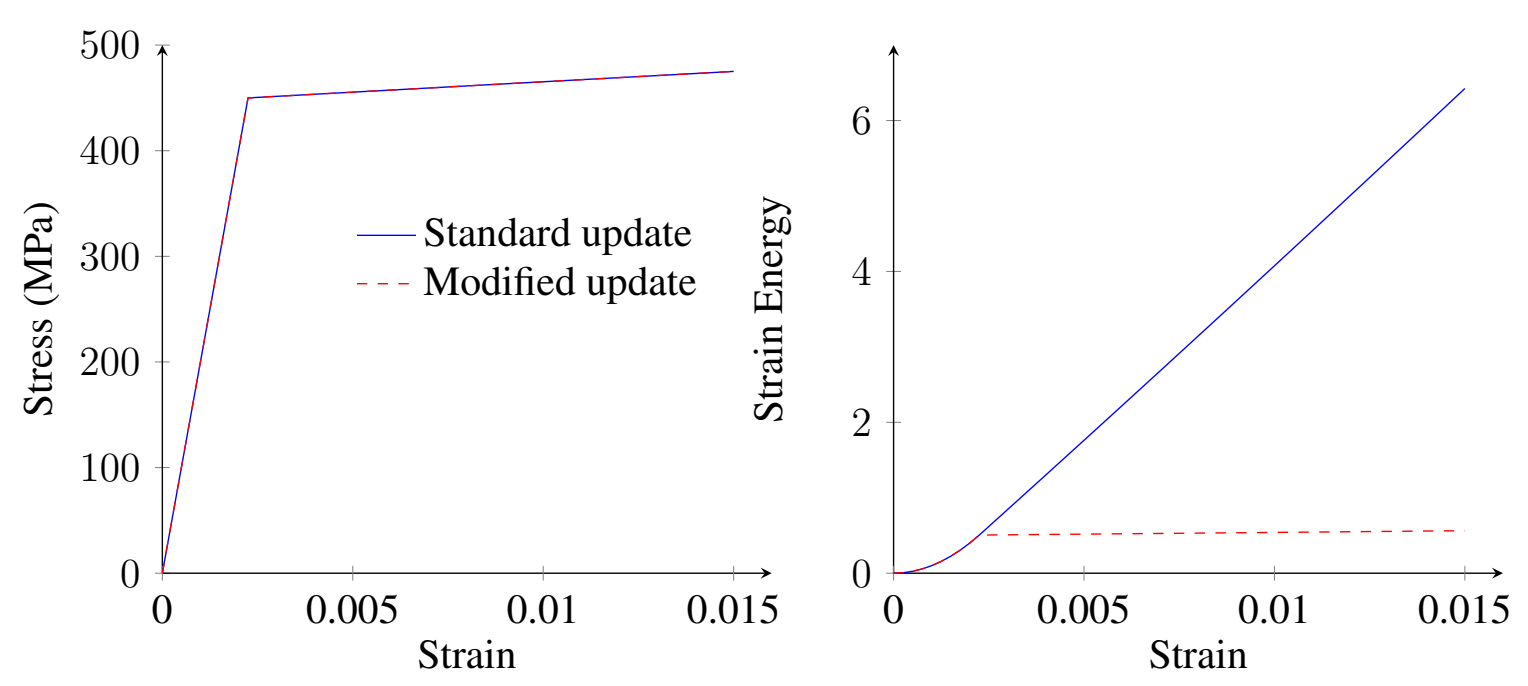

Figure 24: Plots of stress and strain energy response from a uniaxial with linear hardening calculation. The standard update plots refer to the update from the algorithm shown in Box 1 and the Modified update plots refer to the update from the algorithm summarized in Section 4.1.1. The stress plots are indistinguishable while the strain energy plots are clearly different.

algorithm described in Box 1 and the Modified update refers to computations performed using the algorithm summarized in Section 4.1.1. It is clear from these plots that the modified algorithm has virtually no effect on the stress. In fact, the difference after 300 load increments is less than $10^{-6}$ percent. The difference in the strain energy calculations, on the other hand, is significant where the standard update obviously produces non-physical results, but the modified update is meaningful.

\section{References}

[1] M. Ambati, T. Gerasimov, and L. De Lorenzis. Phase-field modeling of ductile fracture. Computational Mechanics, 55(5):1017-1040, 2015.

[2] M. Ambati, R. Kruse, and L. De Lorenzis. A phase-field model for ductile fracture at finite strains and its experimental verification. Computational Mechanics, 57(1):149-167, 2016.

[3] L. Ambrosio and V. M. Tortorelli. Approximation of functional depending on jumps by elliptic functional via $\Gamma$-convergence. Communications on Pure and Applied Mathematics, 43(8):999-1036, 1990.

[4] H. Amor, J. J. Marigo, and C. Maurini. Regularized formulation of the variational brittle frac- 
ture with unilateral contact: Numerical experiments. Journal of the Mechanics and Physics of Solids, 57(8):1209-1229, 2009.

[5] Y.-L. Bai and T. Wierzbicki. A new model of metal plasticity and fracture with pressure and Lode dependence. International Journal of Plasticity, 24:1071-1096, 2008.

[6] Y.-L. Bai and T. Wierzbicki. Application of extended mohr-coulomb criterion to ductile fracture. International Journal of Fracture, 161:1-20, 2010.

[7] Y.-B. Bao and T. Wierzbicki. On fracture locus in the equivalent strain and stress triaxiality space. International Journal of Mechanical Sciences, 46:81-98, 2004.

[8] M. J. Borden, C. V. Verhoosel, M. A. Scott, T. J. R. Hughes, and C. M. Landis. A phasefield description of dynamic brittle fracture. Computer Methods in Applied Mechanics and Engineering, 217-220:77-95, 2012.

[9] M. J. Borden, T. J. R. Hughes, C. M. Landis, and C. V. Verhoosel. A higher-order phasefield model for brittle fracture: Formulation and analysis within the isogeometric analysis framework. Computer Methods in Applied Mechanics and Engineering, 273:100-118, 2014.

[10] B. Bourdin, G. A. Francfort, and J-J. Marigo. Numerical experiments in revisited brittle fracture. Journal of the Mechanics and Physics of Solids, 48(4):797-826, 2000.

[11] B. Bourdin, G. A. Francfort, and J-J. Marigo. The variational approach to fracture. Journal of Elasticity, 91(1-3):5-148, 2008.

[12] B. Bourdin, C. Larsen, and C. Richardson. A time-discrete model for dynamic fracture based on crack regularization. International Journal of Fracture, 168(2):133-143, 2011.

[13] Blaise Bourdin, Chukwudi Paul Chukwudozie, and Keita Yoshioka. A variational approach to the numerical simulation of hydraulic fracturing. In Proceedings of the 2012 SPE Annual Technical Conference and Exhibition, volume SPE 159154. Society of Petroleum Engineers, 2012. doi: 10.2118/159154-MS.

[14] P. Bridgman. Studies in Large Plastic Flow and Fracture. McGraw-Hill Book Company, London, 1952.

[15] S. Burke, C. Ortner, and E. Süli. An adaptive finite element approximation of a generalized ambrosio-tortorelli functional. Mathematical Models \& Methods in Applied Sciences, 23(9): 1663 - 1697, 2013. 
[16] G. A. Francfort and J-J. Marigo. Revisiting brittle fracture as an energy minimization problem. Journal of the Mechanics and Physics of Solids, 46(8):1319-1342, 1998.

[17] A. Ghahremaninezhad and K. Ravi-Chandar. Ductile failure behavior of polycrystalline al 6061-t6. International Journal of Fracture, 174(2):177-202, 2012.

[18] A.L. Gurson. Continuum theory of ductile rupture by void nucleation and growth - part I. Yield criteria and flow rules for porous ductile media. Journal of Engineering Materials and Technology, 99:2-15, 1977.

[19] M. E. Gurtin. Generalized Ginzburg-Landau and Cahn-Hilliard equations based on a microforce balance. Physica D: Nonlinear Phenomena, 92(3-4):178-192, 1996.

[20] S.S. Halton, S. Kyriakides, and K. Ravi-Chandar. Ductile failure under combined shear and tension. International Journal of Solids and Structures, 50:1507-1522, 2013.

[21] M. Hofacker and C. Miehe. A phase field model of dynamic fracture: Robust field updates for the analysis of complex crack patterns. International Journal for Numerical Methods in Engineering, 93(3):276-301, 2013.

[22] T. J. R. Hughes. The Finite Element Method: Linear Static and Dynamic Finite Element Analysis. Dover Publications, Mineola, NY, 2000.

[23] T. J. R. Hughes, J. A. Cottrell, and Y. Bazilevs. Isogeometric analysis: CAD, finite elements, NURBS, exact geometry and mesh refinement. Computer Methods in Applied Mechanics and Engineering, 194:4135-4195, 2005.

[24] G.R. Johnson and W.H. Cook. Fracture characteristics of three metals subjected to various strains, strain rates, temperatures and pressures. Engineering Fracture Mechanics, 21:31-48, 1985.

[25] C. J. Larsen. Models for dynamic fracture based on griffith's criterion. In K. Hackl, editor, IUTAM Symposium on Variational Concepts with Applications to the Mechanics of Materials, volume 21, pages 131-140. Springer Netherlands, 2010.

[26] C. J. Larsen, C. Ortner, and E. Süli. Existence of solutions to a regularized model of dynamic fracture. Mathematical Methods and Models in Applied Sciences, 20(7):1021-1048, 2010.

[27] Colin McAuliffe and Haim Waisman. A unified model for metal failure capturing shear banding and fracture. International Journal of Plasticity, 65:131 - 151, 2015. 
[28] F.A. McClintock. A criterion for ductile fracture by the growth of holes. Journal of Applied Mechanics, 35:363-371, 1968.

[29] C. Miehe, M. Hofacker, and F. Welschinger. A phase field model for rate-independent crack propagation: Robust algorithmic implementation based on operator splits. Computer Methods in Applied Mechanics and Engineering, 199(45-48):2765-2778, 2010.

[30] C. Miehe, F. Welschinger, and M. Hofacker. Thermodynamically consistent phase-field models of fracture: Variational principles and multi-field FE implementations. International Journal for Numerical Methods in Engineering, 83(10):1273-1311, 2010.

[31] C. Miehe, M. Hofacker, L.-M. Schänzel, and F. Aldakheel. Phase field modeling of fracture in multi-physics problems. Part II. coupled brittle-to-ductile failure criteria and crack propagation in thermo-elastic-plastic solids. Computer Methods in Applied Mechanics and Engineering, 294:486 - 522, 2015.

[32] Christian Miehe and Lisa-Marie Schänzel. Phase field modeling of fracture in rubbery polymers. part i: Finite elasticity coupled with brittle failure. Journal of the Mechanics and Physics of Solids, 65:93 - 113, 2014.

[33] Christian Miehe, Lisa-Marie Schänzel, and Heike Ulmer. Phase field modeling of fracture in multi-physics problems. part i. balance of crack surface and failure criteria for brittle crack propagation in thermo-elastic solids. Computer Methods in Applied Mechanics and Engineering, 294:449 - 485, 2015.

[34] Christian Miehe, Fadi Aldakheel, and Arun Raina. Phase field modeling of ductile fracture at finite strains. a variational gradient-extended plasticity-damage theory. International Journal of Plasticity, In press, doi:10.1016/j.jplas.2016.04.011, 2016.

[35] Andro Mikelic̀, Mary F. Wheeler, and Thomas Wick. A phase-field method for propagating fluid-filled fractures coupled to a surrounding porous medium. Multiscale Modeling \& Simulation, 13:367-398, 2015.

[36] D. Mumford and J. Shah. Optimal approximations by piecewise smooth functions and associated variational problems. Communications on Pure and Applied Mathematics, 42(5): 577-685, 1989.

[37] T Pardoen, Y Marchal, and F Delannay. Thickness dependence of cracking resistance in thin aluminium plates. Journal of the Mechanics and Physics of Solids, 47(10):2093-2123, 1999. 
[38] K. Pham, H. Amor, J-J. Marigo, and C. Maurini. Gradient damage models and their use to approximate brittle fracture. International Journal of Damage Mechanics, 20(4):618-652, 2011.

[39] J.R. Rice and D.M. Tracey. On the ductile enlargement of voids in triaxial stress fields. Journal of the Mechanics and Physics of Solids, 17:201-217, 1696.

[40] D. Schillinger, L. Dedè, M. A. Scott, J. A. Evans, M. J. Borden, E. Rank, and T. J. R. Hughes. An isogeometric design-through-analysis methodology based on adaptive hierarchical refinement of NURBS, immersed boundary methods, and T-spline CAD surfaces. Computer Methods in Applied Mechanics and Engineering, 249-252:116-150, 2012.

[41] M. A. Scott, D. C. Thomas, and E. J. Evans. Isogeometric spline forests. Computer Methods in Applied Mechanics and Engineering, 269(0):222-264, 22014.

[42] J. C. Simo. A framework for finite strain elastoplasticity based on maximum plastic dissipation and the multiplicative decomposition: Part I. continuum formulation. Computer Methods in Applied Mechanics and Engineering, 66(2):199 - 219, 1988.

[43] J. C. Simo and T. J. R. Hughes. Computational Inelasticity. Springer-Verlag, New York, 1998.

[44] H. Stumpf and K. Hackl. Micromechanical concept for the analysis of damage evolution in thermo-viscoelastic and quasi-brittle materials. International Journal of Solids and Structures, 40(6):1567-1584, 2003.

[45] V. Tvergaard and A. Needleman. Analysis of the cup-cone fracture in a round tensile bar. Acta Metallurgica, 32:157-169, 1984.

[46] K. G. Webster. Investigation of close proximity underwater explosition effects on a shiplike structure using the multi-material arbitrary Lagrangian Eulerian finite element method. Master's thesis, Virginia Polytechnic Institute and State University, January 2007.

[47] Z.A. Wilson, M. J. Borden, and C. M. Landis. A phase-field model for fracture in piezoelectric ceramics. International Journal of Fracture, 183(2):135-153, 2013. 